\title{
Norm-induced Densities and Testing the Boundedness of a Convex Set
}

\author{
Alexandre Belloni \\ MIT Operations Research Center, E40-129, 77 Massachusetts Ave., Cambridge, Massachusetts 02142, USA \\ email: belloni@mit.edu http://web.mit.edu/belloni/www
}

In this paper we explore properties of a family of probability density functions, called norm-induced densities, defined as

$$
f_{t}(x)= \begin{cases}\frac{e^{-t\|x\|^{p}} d x}{\int_{K} e^{-t\|y\|^{p}} d y}, & x \in K \\ 0, & x \notin K,\end{cases}
$$

where $K$ is a $n$-dimensional convex set, parameters $t>0$ and $p>0$, and $\|\cdot\|$ is any norm. We also develop connections between these densities and geometric properties of $K$ such as diameter, width of the recession cone, and others.

Moreover, we establish a new set inclusion characterization for convex sets. This leads to a new concentration phenomena for unbounded convex sets. More explicitly, we show that most points of a convex set are contained in any enlargement of the set's recession cone.

Finally, these properties are used to develop an efficient probabilistic algorithm to test whether a convex set, represented only by a membership oracle, is bounded or not, where the algorithm reports an associated certificate of boundedness or unboundedness.

Key words: convex sets ; unbounded sets ; homotopy ; random walk ; membership oracle

MSC2000 Subject Classification: Primary: 52A20 , 90C60 ; Secondary: 90C25

OR/MS subject classification: Primary: Convexity ; Secondary: Random Walk

1. Introduction. The geometry of convex sets has been extensively studied during the past halfcentury. More recently, the interplay between convex geometry and probability theory has been investigated. Among many possible research directions, log-concave probability measures provide an interesting framework that generalizes uniform densities on convex sets but preserves many interesting properties, see [1]. Here we will focus on a family of densities functions whose properties relate to geometric properties of the convex set defined by its support. We say that a probability density function $f_{t}: \mathbb{R}^{n} \rightarrow \mathbb{R}_{+}$is called norm-induced if

$$
f_{t}(x)= \begin{cases}\frac{e^{-t\|x\|^{p}} d x}{\int_{K} e^{-t\|y\|^{p}} d y}, & x \in K \\ 0, & x \notin K\end{cases}
$$

where $K$ is a convex set in $\mathbb{R}^{n}, t>0$ and $p>0$ are parameters, and $\|\cdot\|$ is a fixed norm. Note that a probability density function of the form (1) is always proper. Moreover, $f_{t}$ is log-concave only if $p \geq 1$.

We will explore the connection between these densities and geometric properties of the convex set $K$ itself, usually as a function of the parameter $t$. The geometry of unbounded convex sets plays an important role in our analysis. For instance, given an arbitrary unbounded convex set we show that most of its points are contained in any enlargement of its recession cone. This simple geometric phenomenon motivates many of our results.

In the case of $p \geq 1$, a random variable whose density distribution function is $f_{t}$ can be efficiently simulated (at least approximately) by geometric random walk algorithms [10. In turn, theoretical results on $f_{t}$ can be used to construct (implementable) algorithms to test properties of $K$ itself.

We also develop an algorithm to test if a given convex set $K \subset \mathbb{R}^{n}$ is bounded or unbounded. In either case the algorithm will construct an associated certificate of boundedness or unboundedness based on the properties established in Section 3. We emphasize that algorithms for this problem are closely related to the representation used to describe the set $K$. Our interest lies in cases in which the convex set is given only by a membership oracle. This (minimal assumption) framework is the standard framework in several applications in the computer science literature. Furthermore, it covers many other problems of interest such as convex feasibility.

The decision problem of testing for boundedness has a variety of interesting consequences. In recent years, several probabilistic methods have been proposed to compute quantities like centroid, volume 
[13, convex optimization [6, and many others [1], in the context of convex bodies. In all these cases, boundedness of a convex set is a fundamental assumption for whose testing our algorithm provides a constructive approach. Khachiyan established the equivalence between a strongly polynomial algorithm for linear programming and a strongly polynomial algorithm for testing unboundedness of a convex set associated with a system of linear inequalities [7. Moreover, linear homogeneous conic feasibility problems of the form

$$
\left\{\begin{array}{rll}
A x & \in & C \\
x & \in & \mathbb{R}^{n} \backslash\{0\}
\end{array}\right.
$$

(where $C$ is closed convex cone) can be converted into our framework by defining

$$
K=\left\{x \in \mathbb{R}^{n}: A x+h \in C\right\}
$$

for any $h \in \operatorname{int} C$. In this case, $0 \in \operatorname{int} K$, and the recession cone of $K$ coincides with the set of feasible solutions of the original system (2). Moreover, $K$ is bounded only if (2) is infeasible. Finally, a membership oracle for the cone $C$ suffices to construct a membership oracle for $K$ and its recession cone $C_{K}$.

The implementability of our algorithm relies on the ability to sample random variables distributed according to a probability density $f_{t}$. Over the last decade many important developments on sampling from log-concave densities, most notably via geometric random walks, have been observed. In particular, the hit-and-run random walk has been extensively analyzed and polynomial rates of convergence have been established for this particular random walk under the log-concavity assumption [8, 11, 12, 10. Besides, the homotopy analysis proposed here is similar to the analysis done by Lovász and Vempala in 13] of the algorithm they called reverse annealing, which was applied to the problem of computing the volume of a (bounded) convex body. However, our approach differs from 13 with in respects: by using a different density family, and by dealing explicitly with the possible unboundedness of $K$.

In the presence of additional structure, other algorithms are available in the literature. For example, assuming that a self-concordant barrier function is available for $K$, minimizing such function leads to appropriate certificates of boundedness or unboundedness (note that the minimum is finite only if $K$ is bounded). That idea was used first by de Ghellink and Vial in [3. for linear programming and more recently by Nesterov, Todd and Ye 14 for nonlinear programming problems. Moreover, if $K$ is given explicitly by a set of linear inequalities, one can identify an element of the recession cone by solving a linear programming problem.

We emphasize that none of these approaches extends to the membership oracle framework. In fact, negative results do exist for approximating the diameter of a bounded convex set, which is a closely related problem. Lovász and Simonovits [9] show that no polynomial time algorithm (deterministic or probabilistic) can approximate the diameter of a convex set within a factor of $\sqrt{n}$ in polynomial time under the membership oracle framework. Thus, it is notable that, as we show, testing if a convex set is unbounded is solvable in polynomial time.

An outline of this paper is as follows. Section 2 illustrates the geometric intuition underlying many results. Then we establish many properties relating the density functions (1) and the convex set $K$ in Section 3. The algorithm to test boundedness is presented in Section 4 and its analysis is presented in the following sections. Finally, Appendix A contains the details on how to implement the hit-and-run geometric random walk efficiently for the density functions used in the algorithm.

1.1 Preliminaries, definitions, and notation. Recall that a real-valued function $\|\cdot\|: \mathbb{R}^{n} \rightarrow \mathbb{R}_{+}$ is said to be a norm if:

(i) $\|x\|=0$ only if $x=0$;

(ii) $\|t x\|=|t|\|x\|$ for any $t \in \mathbb{R}$;

(iii) $\|x+y\| \leq\|x\|+\|y\|$ for any $x, y \in \mathbb{R}^{n}$.

For a given norm, we can define a unit ball

$$
B_{\|\cdot\|}(x, r)=B(x, r)=\left\{y \in \mathbb{R}^{n}:\|y-x\| \leq r\right\},
$$

and a unit sphere $S^{n-1}=\left\{y \in \mathbb{R}^{n}:\|y\|=1\right\}$. 
The Euclidean inner product is denoted by $\langle\cdot, \cdot\rangle$ and $\|\cdot\|_{2}=\sqrt{\langle\cdot, \cdot\rangle}$ denotes the Euclidean norm induced by it. For $x \in \mathbb{R}^{n}$ and $r>0$, let $B_{2}(x, r)$ denote the Euclidean ball centered at $x$ with radius $r$, i.e., $B_{2}(x, r)=\left\{y \in \mathbb{R}^{n}:\|x-y\|_{2} \leq r\right\}$. The unit Euclidean sphere of $\mathbb{R}^{n}$ is denoted by $S_{2}^{n-1}$, i.e., $S_{2}^{n-1}=\left\{y \in \mathbb{R}^{n}:\|y\|=1\right\}$.

The dual norm of $\|\cdot\|$ induced by $\langle\cdot, \cdot\rangle$ is defined by

$$
\|s\|_{*}=\max \{\langle s, x\rangle: x \in B(0,1)\},
$$

for which we can also define a unit ball $B_{*}(s, r)=\left\{w \in \mathbb{R}^{n}:\|w-s\|_{*} \leq r\right\}$ and a unit sphere $S_{*}^{n-1}$. By definition we have that $|\langle s, x\rangle| \leq\|s\|_{*}\|x\|$. The dual norm completely defines the original norm, since we have

$$
\|x\|=\max \left\{\langle s, x\rangle: s \in B_{*}(0,1)\right\} .
$$

That is, the dual norm of the dual norm is the original norm. Recall that the dual norm of the Euclidean norm is also the Euclidean norm, which is said to be self-dual.

A set $K$ is convex if $x, y \in S$ implies $\alpha x+(1-\alpha) y \in S$ for every $\alpha \in[0,1]$. $C$ is a cone if $x \in C$ implies $\alpha x \in C$ for every $\alpha \geq 0$. If $C$ is a convex cone, the width of $C$ is given by

$$
\tau_{C}=\max _{x, \tau}\{\tau: B(x, \tau) \subset C,\|x\|=1\},
$$

the radius of the largest ball contained in $C$ centered at a unit vector (in the appropriate norm). $L$ is a subspace of $\mathbb{R}^{n}$ if $x, y \in L$ implies that $\alpha x+\beta y \in L$ for every $\alpha, \beta \in \mathbb{R}$.

For a set $S$, the operations $\operatorname{conv}(S), \operatorname{cone}(S), \operatorname{ext}(S), \operatorname{int}(S), \operatorname{cl}(S), \operatorname{diam}(S)$, and $\operatorname{Vol}(S) \operatorname{denote}$, respectively, the convex hull, conic hull, extreme points, interior, closure, diameter, and volume of $S$ (see 15 for complete definitions). Also, for $x \in \mathbb{R}^{n}$, let $\operatorname{dist}(x, S)=\inf \{\|x-y\|: y \in S\}$ denote the distance from $x$ to $S$. For a scalar $u$, set $(u)_{+}=\max \{0, u\}$, and for a matrix $M$ denote by $\lambda_{\max }(M)$ (respectively $\left.\lambda_{\min }(M)\right)$ its maximum (respectively minimum) eigenvalue.

A membership oracle for a set $S$ is any algorithm, that given any point $x \in \mathbb{R}^{n}$, correctly identifies if $x \in S$ or not. Let $1_{S}$ denote the indicator function of the set $S$, that is, $1_{S}(x)=1$ if $x \in S$ and $1_{S}(x)=0$ otherwise.

With respect to complexity notation, $g(n)$ is said to be $O(f(n))$ if there exists a constant $M$ such that $g(n) \leq M f(n)$, while $g(n, \mu)$ is $O^{*}(f(n, \mu))$ if there exists constants $M$ and $k$ such that $g(n, \mu) \leq$ $M f(n, \mu) \ln ^{k} n$ (that is, the $O^{*}$ notation omit logarithmic factors of the dimension but still shows the dependence on other possible condition measure $\mu$ ).

1.2 Logconcave densities: concepts and notation. We define $\pi_{f}$ as the probability measure associated with a probability density function $f\left(\right.$ i.e, $\left.\pi_{f}(S)=\int_{S} f(x) d x\right), E_{f}[\cdot]$ as the expectation with respect to $f$, and $z_{f}$ as the mean of a random variable whose probability density function is $f$. The following class of functions plays a central role in the sampling literature.

Definition 1.1 A function $f: \mathbb{R}^{n} \rightarrow \mathbb{R}_{+}$is logconcave if for any two points $x, y \in \mathbb{R}^{n}$ and any $\lambda \in(0,1)$,

$$
f(\lambda x+(1-\lambda) y) \geq f(x)^{\lambda} f(y)^{1-\lambda} .
$$

Definition 1.1 implies that $\ln f$ is a concave function and, in particular, the support of $f$ is a convex set. We say that a random variable is logconcave if its probability density function is a logconcave function. Gaussians, exponential and uniforms densities are examples of logconcave densities.

There are a variety of metrics available for probability densities. Here, we will make use of two of them: the total variation norm, defined as

$$
\|f-g\|_{T V}=\frac{1}{2} \int_{\mathbb{R}^{n}}|f(x)-g(x)| d x
$$

and the $L^{2}$ norm of $f$ with respect to $g$, defined as

$$
\|f / g\|=E_{f}\left[\frac{f(x)}{g(x)}\right]=\int_{\mathbb{R}^{n}} \frac{f(x)}{g(x)} f(x) d x=\int_{\mathbb{R}^{n}}\left(\frac{f(x)}{g(x)}\right)^{2} g(x) d x .
$$

The following useful concept is associated with the covariance matrix induced by $f$. 


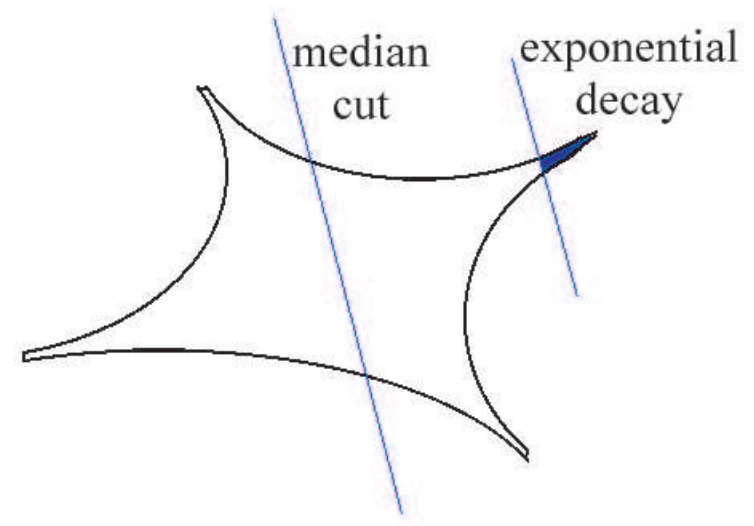

Figure 1: High-dimensional bounded convex sets and volume. Exponential decay as we move away the median cut.

Definition $1.2 A$ density function $f$ with expectation $z_{f}$ is said to be $C$-isotropic if for every vector $v$,

$$
\frac{\|v\|_{2}^{2}}{C} \leq \int_{\mathbb{R}^{n}}\left\langle v, x-z_{f}\right\rangle^{2} f(x) d x \leq\|v\|_{2}^{2} C,
$$

equivalently, any eigenvalue $\lambda$ of the covariance matrix of $f$ satisfies: $\frac{1}{C} \leq \lambda \leq C$.

A function $f$ is said to be in isotropic position if it is 1-isotropic, that is, its covariance matrix is the identity. Thus, any density can be made isotropic by a linear transformation of the space.

2. $\epsilon$-Enlargements of the Recession Cone. In this section we revisit a classical representation theorem for closed convex sets and we provide a new set inclusion characterization for such sets which will be key in our analysis.

Let $K \subset \mathbb{R}^{n}$ be a closed convex set. As a matter of convenience, assume $K$ is full dimensional, as one can always work within the affine hull of $K$, but at considerable notational and expositional expense. For the sake of exposition, in this section we will assume that $K$ contains no lines (in the upcoming sections we do not make such assumption).

As is standard in convex theory, the set of all directions of half-lines contained in $K$ defines the recession cone of $K$ denoted by $C_{K}$, i.e.,

$$
C_{K}=\left\{d \in \mathbb{R}^{n}: K+d \subseteq K\right\},
$$

which is a closed convex cone ([15] Corollary 8.3.2). Moreover, it is well-known that $K$ is unbounded only if $C_{K} \neq\{0\}([15]$ Theorem 8.4).

Under this framework, $K$ contains at least one extreme point ([15] Corollary 18.5.3). Thus, the following representation theorem for closed, line-free, convex sets applies to $K$.

THEOREM 2.1 Any closed line-free convex set $K \subset \mathbb{R}^{n}$ can be decomposed into the sum of two sets, the recession cone of $K$ and the convex hull of its extreme points. That is,

$$
K=C_{K}+\operatorname{conv}(\operatorname{ext}(K))
$$

In order to develop intuition on the relation between high-dimensional convex sets and volume, we need to understand how to draw pictures of what high-dimensional convex sets look like. The intuition for convex bodies (bounded convex sets with nonempty interior) was first suggested by Gromov and Milman in [4. The fact that the volume of parallel intersections of half-spaces with $K$ decays exponentially fast after passing the median level must be taken into account. As suggested by Gromov and Milman, small dimensional pictures of a high-dimensional convex body should have a hyperbolic form, see Figure 1 . 


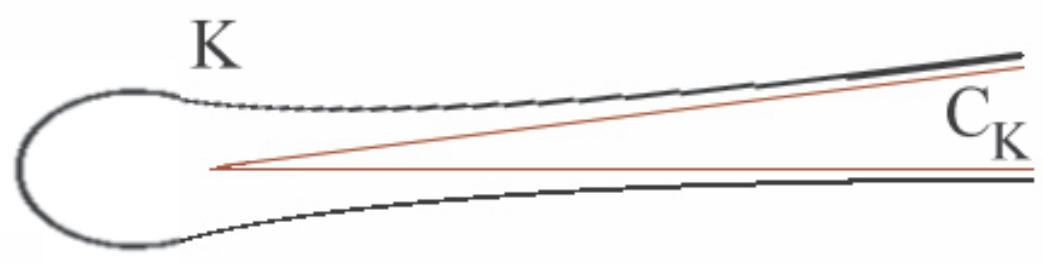

Figure 2: High-dimensional unbounded convex sets and volume. Most of the mass concentrates around the recession cone.

However, our concern here is to extend such intuition to unbounded convex sets. In this context a similar (concentration) phenomenon will also be observed. Assuming that the recession cone has positive width, "most of the points" of the set are in the recession cone. (Note that one needs to be careful when quantifying "most of the points", since the volume is infinite.) Again small dimensional pictures of high-dimensional unbounded convex sets must have a hyperbolic form, see Figure 2.

In fact, even if the recession cone has zero width, "most of the points" of $K$ will be contained in any $\epsilon$-enlargement of the recession cone, where the latter is formally defined as:

$$
C_{K}^{\epsilon}=\text { cone }\left\{S^{n-1} \cap\left(C_{K}+B(0, \epsilon)\right)\right\} .
$$

The following properties of the $\epsilon$-enlargement of the recession cone follow directly from the definition.

LEMMA 2.1 For $\epsilon>0$ sufficiently small, we have that:

(i) $C_{K}^{\epsilon}$ is a closed cone and contains no line;

(ii) if $C_{K}=\{0\}$, then $C_{K}^{\epsilon}=\{0\}$;

(iii) if $C_{K} \neq\{0\}$, then $\tau_{C_{K}^{\epsilon}} \geq \max \left\{\tau_{C_{K}}, \epsilon / 3\right\}$.

Proof. $\quad C_{K}^{\epsilon}$ is a closed cone by definition. Since $C_{K}$ is a pointed cone, the Pompeiu-Hausdorff distance of the cone $C_{K}$ to any cone $C$ that contains a line is bounded away from zero, $\delta\left(C_{K}, C\right)=$ $\sup _{\|z\| \leq 1}\left|\operatorname{dist}\left(z, C_{K}\right)-\operatorname{dist}(z, C)\right|>c>0$ (see [5] for details). Moreover, $\delta\left(C_{K}, C_{K}^{\epsilon}\right) \leq \epsilon$. Thus we can choose $\epsilon<c$ and $C_{K}^{\epsilon}$ does not contain lines.

If $C_{K}=\{0\}$, for $\epsilon<1$ we have $S^{n-1} \cap\left(C_{K}+B(0, \epsilon)\right)=\emptyset$ and $C_{K}^{\epsilon}=\{0\}$ and (ii) follows.

To prove (iii), observe that $C_{K} \subset C_{K}^{\epsilon}$ implies that $\tau_{C_{K}^{\epsilon}} \geq \tau_{C_{K}}$. Moreover, if $z \in C_{K},\|z\|=1$, we will show that $B(z, \epsilon / 3) \subset C_{K}^{\epsilon}$. Take $x \in B(z, \epsilon / 3)$ and let $w:=x /\|x\|$. Since $\|z\|=1$, we have $1+\epsilon / 3 \geq\|x\| \geq 1-\epsilon / 3$. Then

$$
\begin{aligned}
\left\|\frac{x}{\|x\|}-z\right\| & =\left\|\frac{x-z}{\|x\|}+\frac{z}{\|x\|}-z\right\| \leq \frac{\|x-z\|}{\|x\|}+\|z\|\left(\frac{1}{\|x\|}-1\right) \\
& \leq \frac{\epsilon}{3} \frac{1}{1-\epsilon / 3}+\frac{1}{1-\epsilon / 3}-1=\frac{2 \epsilon}{3-\epsilon}<\epsilon,
\end{aligned}
$$

where the last inequality follows since $\epsilon<1$. Since $x /\|x\| \in S^{n-1}$ and $x /\|x\| \in B(z, \epsilon), x \in C_{K}^{\epsilon}$. This implies that $\tau_{C_{K}^{\epsilon}} \geq \epsilon / 3$.

Now we are in position to obtain a set inclusion characterization of the aforementioned geometric phenomena, which will motivate most of the analysis in the sections to come.

THEOREM 2.2 Let $K$ be a convex set. Then, for any $\epsilon>0$, there exists a positive scalar $R_{\epsilon}$ such that

$$
K \subseteq C_{K}^{\epsilon}+B\left(0, R_{\epsilon}\right)
$$


Proof. Without loss of generality, assume $0 \in K$. Suppose that there exists a sequence $\left\{x^{j}\right\}$ of elements of $K$ such that $\operatorname{dist}\left(x^{j}, C_{K}^{\epsilon}\right)>j$. The normalized sequence has a convergent subsequence, $\left\{d^{k_{j}}=x^{k_{j}} /\left\|x^{k_{j}}\right\|\right\}$, to a point $d$. Since $K$ is convex, closed and $0 \in K, d^{k_{j}}$ and $d$ are in $K$. In fact, $d \in C_{K}$. For any $\epsilon>0$, there exists a number $j_{0}$ such that

$$
\left\|d^{k_{j}}-d\right\|<\epsilon \text { for all } j>j_{0} \text {. }
$$

Therefore, we have $d^{k_{j}} \in C_{K}^{\epsilon}$ for $j>j_{0}$. In addition, $x^{k_{j}} \in C_{K}^{\epsilon}$, since $C_{K}^{\epsilon}$ is a cone, contradicting $\operatorname{dist}\left(x^{j}, C_{K}^{\epsilon}\right)>j$ for every $j$.

It is well-known that for the polyhedral case Theorem 2.2 holds with $\varepsilon=0$ but this is not the case in general.

ExAmple 2.1 Consider $K=\left\{(x, y) \in \mathbb{R}^{2}: x>-1, \ln (x+1) \geq y\right\}$. Here the recession cone is $C_{K}=$ $\mathbb{R}_{+} \times \mathbb{R}_{-}$and $\tau_{C_{K}}=1 / \sqrt{2}$. Note that for all $R>1$, the point $(R, \ln R) \in K$ and $\operatorname{dist}\left((R, \ln R), C_{K}\right)=$ $\ln R$. Consider $\epsilon<1 / 5$ which implies that $C_{K}^{\epsilon}$ contains no lines. For any point $(x, y) \in K$ such that $x \geq \frac{1}{\varepsilon} \ln ^{2} \frac{1}{\varepsilon}$, we have that $y \leq \ln x \leq \epsilon x$ which implies that $(x, y) \in C_{K}^{\epsilon}$. Thus, Theorem 2.2 holds with $R_{\varepsilon}:=\frac{2}{\varepsilon} \ln ^{2} \frac{1}{\varepsilon}$

The $\epsilon$-enlargement of the recession cone captures all points of $K$ except for a bounded subset. Thus, assuming that $K$ is unbounded, such geometric phenomenon implies that "most points" of $K$ will be contained in $K \cap C_{K}^{\epsilon}$. Moreover, if $\tau_{C_{K}}>0$, most points of $K$ will actually be contained in $C_{K}$ itself. This is quantified in the next results.

Lemma 2.2 Let $C_{K}$ be a convex cone with strictly positive width $\tau_{C_{K}}$. Then

$$
\operatorname{Vol}_{n-1}\left(C_{K}^{\epsilon} \cap S^{n-1}\right) \leq\left(1+\frac{2 \epsilon}{\tau_{C_{K}}}\right)^{n} \operatorname{Vol}_{n-1}\left(C_{K} \cap S^{n-1}\right) .
$$

Proof. By definition of $\tau_{C_{K}}, B\left(z, \tau_{C_{K}}\right) \subset C_{K}$ for some $z \in S^{n-1}$. Since $C_{K}$ and $C_{K}^{\epsilon}$ are cones,

$$
\frac{\operatorname{Vol}_{n-1}\left(C_{K}^{\epsilon} \cap S^{n-1}\right)}{\operatorname{Vol}_{n-1}\left(C_{K} \cap S^{n-1}\right)}=\frac{\operatorname{Vol}\left(C_{K}^{\epsilon} \cap B(0,1)\right)}{\operatorname{Vol}\left(C_{K} \cap B(0,1)\right)} \leq \frac{\operatorname{Vol}\left(\left(C_{K} \cap B(0,1)\right)+B(0, \epsilon)\right)}{\operatorname{Vol}\left(C_{K} \cap B(0,1)\right)},
$$

since $\left(C_{K}^{\epsilon} \cap B(0,1)\right) \subset\left(C_{K} \cap B(0,1)+B(0, \epsilon)\right)$. Next, we have $B\left(z / 2, \tau_{C_{K}} / 2\right) \subset\left(C_{K} \cap B(0,1)\right)$, so that

$$
\left(C_{K} \cap B(0,1)\right)+B(0, \epsilon) \subset\left(C_{K} \cap B(0,1)\right)+\frac{2 \epsilon}{\tau_{C_{K}}}\left(C_{K} \cap B(0,1)-\frac{z}{2}\right),
$$

and the result follows.

THEOREM 2.3 Let $X_{t}$ be norm-induced random variable defined by a norm $\|\cdot\|$, parameters $t>0$ and $p>0$, and a convex set $K$. Moreover, let $Y_{t}$ also be a norm-induced random variable defined by the same norm $\|\cdot\|$, the same parameters $t>0$ and $p>0$, but with a different convex set $K_{Y}$ such that

Then

$$
K \subseteq K_{Y} \subseteq C_{K}^{\varepsilon}+B\left(0, R_{\varepsilon}\right)
$$

$$
P\left(X_{t} \in C_{K}\right) \geq P\left(Y_{t} \in C_{K}\right) \geq\left(1-\frac{2 \epsilon}{\tau_{C_{K}}}\right)^{n} P\left(Y_{t} \in C_{K}^{\varepsilon}\right) .
$$

Proof. By definition we have that

$$
P\left(Y_{t} \in C_{K}\right)=P\left(Y_{t} \in C_{K} \mid Y_{t} \in K\right) P\left(Y_{t} \in K\right)+P\left(Y_{t} \in C_{K} \mid Y_{t} \notin K\right) P\left(Y_{t} \notin K\right) .
$$

Next, note that $P\left(Y_{t} \in C_{K} \mid Y_{t} \notin K\right)=0$ and $P\left(Y_{t} \in C_{K} \mid Y_{t} \in K\right)=P\left(X_{t} \in C_{K}\right)$ since $C_{K} \subset K \subset K_{Y}$. These relations imply the first inequality.

For simplicity, let $A=\left\{x \in \mathbb{R}^{n}:\|x\|=\rho\right\}$ denote the sphere of radius $\rho$. To prove the second inequality, note that for any $\rho \geq 0$

$$
\begin{aligned}
P\left(Y_{t} \in C_{K} \mid\left\|Y_{t}\right\|=\rho\right) & =\frac{\operatorname{Vol}_{n-1}\left(C_{K} \cap A\right)}{\operatorname{Vol}_{n-1}(S \cap A)} \geq\left(1-\frac{2 \epsilon}{\tau_{C_{K}}}\right)^{n} \frac{\mathrm{Vol}_{n-1}\left(C_{K}^{\varepsilon} \cap A\right)}{\operatorname{Vol}_{n-1}(S \cap A)} \\
& \geq\left(1-\frac{2 \epsilon}{\tau_{C_{K}}}\right)^{n} \frac{\mathrm{Vol}_{n-1}\left(C_{K}^{\varepsilon} \cap A \cap S\right)}{\operatorname{Vol}_{n-1}(S \cap A)} \\
& =\left(1-\frac{2 \epsilon}{\tau_{C_{K}}}\right)^{n} P\left(Y_{t} \in C_{K}^{\epsilon} \mid\left\|Y_{t}\right\|=\rho\right)
\end{aligned}
$$


where the first inequality follows from Lemma 2.2 and the second because $C_{K}^{\varepsilon}$ might not be contained in $S$. Since this holds for every $\rho \geq 0$, the result follows.

Taken together, Theorems 2.2 and 2.3 with $\epsilon \leq \tau_{C_{K}} / 4 n$ imply that we have at least a constant fraction (independent of the dimension) of the points in $C_{K}^{\epsilon}$ in $C_{K}$. Thus, finding points in $C_{K}$ is easily achieved by finding random points in $C_{K}^{\epsilon} \cap K$.

3. Geometry of norm-induced densities. In this section we establish several properties of the random variable $X_{t}$, whose density function $f_{t}$ is given by (1). Many properties of $X_{t}$ can be related with a variety of geometric quantities/properties of the set $K$ itself. These results are partially motivated by known properties of uniform distributions over convex sets. Nonetheless, norm-induced densities may fail to be log-concave which is a key property used in similar results [11. Moreover, for algorithmic reasons, we are also interested in relating explicitly the dependence between geometric quantities of $K$ and the parameter $t$ of the norm-induced density. For the sake of exposition, we will assume the following:

ASSUMPtion $3.1 \mathrm{~K}$ is a closed convex set that contains the origin and has nonempty interior.

Assumption 3.2 There exists a positive number $R$ such that $K \subseteq C_{K}+B(0, R)$.

Assumption 3.1 is needed to ensure that $K$ has positive $n$-dimensional volume (possibly infinite). Nonetheless, one can always work with the affine hull of $K$ if one uses the appropriate lower dimensional volume. As expected, the origin could be replaced by any other point in $K$. All results could be restated for that particular point (if we translate the density $f_{t}$ appropriately).

The focus on convex sets that satisfies Assumption 3.2 is not restrictive. In light of Section 2 we know that such inclusion can always be obtained if the recession cone is properly enlarged. In the case of unbounded convex sets whose recession cone has positive width, Theorem 2.3 shows how to relate the enlarged cone with the original recession cone. On the other hand, for unbounded convex sets whose recession cone has zero width, we know that the recession cone has zero measure for any $f_{t}$. Finally, Assumption 3.2 is always satisfied by any bounded convex set, using $R=\operatorname{diam}(K)$ and $C_{K}=\{0\}$, or any polyhedral convex set.

We start with a simple result of the levels sets of the norm-induced densities.

THEOREM 3.1 The upper level sets of a density function $f_{t}$ defined as in (1). Moreover, consider the function $g(x)=\left\|\nabla \ln f_{t}(x)\right\|_{*}$ which gives the dual norm of the gradient of the logarithm of $f_{t}$ at $x$. This function is well defined even if $f_{t}$ is non-differentiable and we have two cases:

(i) if $p \geq 1$ the lower level sets of $g$ are convex;

(ii) if $0<p \leq 1$ the upper level sets of $g$ are convex.

PROOF. The upper level sets of $f_{t}$ are defined by

$$
\left\{x \in K: f_{t}(x) \geq c\right\}=\left\{x \in K: t\|x\|^{p} \leq-\ln \left(c \int f_{t}(z) d z\right)\right\}
$$

. Since the parameters $t$ and $p$ are positive, such set is either a scaling of the unit ball intersected with $K$ or the empty set (both convex sets).

For the second part of the theorem, we first compute $g(x)$. Recall that if $s \in \partial\|x\|(s$ is in the subdifferential of $\|\cdot\|$ at $x)$, we have that $s \in S_{*}^{n-1}$. Then we have

$$
g(x)=\|-t p\| x\left\|^{p-1} s\right\|_{*}=t p\|x\|^{p-1}\|s\|_{*}=t p\|x\|^{p-1} .
$$

The result follows since $g$ is convex for $p \geq 1$ and concave for $0<g \leq 1$.

The next lemma shows that the norm-induced densities exhibit a concentration phenomena similar to log-concave densities. 
Lemma 3.1 Let $X_{t} \sim f_{t}$ as in (1). For $D \geq 4$ we have

$$
P\left(\left\|X_{t}\right\| \geq\left(\frac{n}{t p} D\right)^{1 / p}\right) \leq \frac{1}{3} e^{-\frac{n}{p} D / 3}
$$

Proof. Let $M:=\left(\frac{n}{t p} D\right)^{1 / p}$. Define $K_{Y}:=$ cone $\left\{M S^{n-1} \cap K\right\}$ (a possible non-convex set) and let $Y_{t} \sim e^{-t\|y\|^{p}}$ over $K_{Y}$. Then we have

$$
P\left(\left\|X_{t}\right\| \geq M\right) \leq P\left(\left\|Y_{t}\right\| \geq M\right),
$$

since $\left\{y \in K_{Y}:\|y\| \leq M\right\} \subset K$ and $\{y \in K:\|y\| \geq M\} \subset K_{Y}$.

Then for $Y_{t}$ we have

$$
P\left(\left\|Y_{t}\right\| \geq M\right)=\frac{\int_{y \in K_{Y}:\left\|Y_{t}\right\| \geq M} e^{-t\|y\|^{p}} d y}{\int_{y \in K_{Y}} e^{-t\|y\|^{p}} d y}=\frac{\int_{M}^{\infty} v^{n-1} e^{-t v^{p}} d v}{\int_{0}^{\infty} v^{n-1} e^{-t v^{p}} d v} .
$$

Let $u:=t v^{p}, c:=t M^{p}=\frac{n}{p} D$, and we obtain

$$
P\left(\left\|Y_{t}\right\| \geq M\right)=\frac{\int_{c}^{\infty} u^{\frac{n}{p}-1} e^{-u} d u}{\int_{0}^{\infty} u^{\frac{n}{p}-1} e^{-u} d u} \leq \frac{\int_{c}^{\infty} u^{\left\lceil\frac{n}{p}-1\right\rceil} e^{-u} d u}{\int_{0}^{\infty} u^{\left\lceil\frac{n}{p}-1\right\rceil} e^{-u} d u}=e^{-c} \sum_{i=0}^{\left\lceil\frac{n}{p}-1\right\rceil} \frac{c^{i}}{i !}
$$

Next recall that $i ! \geq(i / e)^{i}$ (from the Stirling's formula, and using the convention that $0^{0}=1$ ) and $\ln (1+h) \leq h$. Then

$$
\begin{aligned}
e^{-c} \sum_{i=0}^{\left\lceil\frac{n}{p}-1\right\rceil} \frac{c^{i}}{i !} & \leq e^{-\frac{n}{p} D} \sum_{i=0}^{\left\lceil\frac{n}{p}-1\right\rceil} \frac{\left(\frac{n}{p} D\right)^{i}}{(i / e)^{i}}=e^{-\frac{n}{p} D} \sum_{i=0}^{\left\lceil\frac{n}{p}-1\right\rceil} e^{i \ln \left(\frac{n / p}{i} D e\right)} \\
& =e^{-\frac{n}{p} D} \sum_{i=0}^{\left\lceil\frac{n}{p}-1\right\rceil} e^{i \ln \left(1+\frac{(n / p)-i}{i}\right)+i \ln (D e)} \leq e^{-\frac{n}{p} D} \sum_{i=0}^{\left\lceil\frac{n}{p}-1\right\rceil} e^{(n / p)-i+i \ln (D e)} \\
& =e^{-\frac{n}{p}(D-1)} \sum_{i=0}^{\left\lceil\frac{n}{p}-1\right\rceil} e^{i \ln (D)}=e^{-\frac{n}{p}(D-1)} \frac{e^{\left\lceil\frac{n}{p}-1\right\rceil \ln (D)}-1}{e^{\ln (D)}-1} \\
& \leq \frac{1}{3} e^{-\frac{n}{p}(D-1-\ln (D))} \leq \frac{1}{3} e^{-\frac{n}{p} D / 3}
\end{aligned}
$$

As anticipated, we will show that the probability of the event $\left\{X_{t} \in C_{K}\right\}$ will be large for "small" values of $t$. To do so, we exploit the spherical symmetry of $f_{t}$ (i.e., $f_{t}(x)=f_{t}(y)$ if $\left.\|x\|=\|y\|\right)$ and the geometric phenomena induced by the representation of Assumption 3.2. That symmetry will allow us to connect the volume of relevant sets with the probability of the event $\left\{X_{t} \in C_{K}\right\}$. Lemma 3.2 below properly quantifies this notion.

Lemma 3.2 Suppose that $K$ is an unbounded convex set, let $X_{t} \sim f_{t}$ as in (1), and let $\rho>6 R / \tau_{C_{K}}$. Then

$$
P\left(X_{t} \in C_{K} \mid\left\|X_{t}\right\|=\rho\right) \geq\left(1-\frac{6 R}{\tau_{C_{K}} \cdot \rho}\right)^{n} .
$$

Proof. Note that restricted on $\left\|X_{t}\right\|=\rho$, the density $f_{t}$ is constant. Thus, we have

$$
P\left(X_{t} \in C_{K} \mid\left\|X_{t}\right\|=\rho\right)=\frac{\operatorname{Vol}_{n-1}\left(C_{K} \cap\left(\rho S^{n-1}\right)\right)}{\operatorname{Vol}_{n-1}\left(K \cap\left(\rho S^{n-1}\right)\right)} .
$$

For any $a, b$, define the sets $K_{[a, b]}:=\{y \in K: a \leq\|y\| \leq b\}$ and $K_{a}:=K_{[a, a]}=\left(a S^{n-1}\right) \cap K$. Set $\bar{\rho}=\rho / 3 \geq 2 R / \tau_{C_{K}}$, and consider the following set inclusions

$$
I_{[\bar{\rho}, \rho]}:=\left\{y \in C_{K}: \bar{\rho} \leq\|y\| \leq \rho\right\} \subset K_{[\bar{\rho}, \rho]} \subset O_{[\bar{\rho}, \rho]}:=\left\{y \in B(0, R)+C_{K}: \bar{\rho} \leq\|y\| \leq \rho\right\} .
$$

We first show that $O_{[\bar{\rho}, \rho]} \subset I_{[\bar{\rho}, \rho]}+B(0,2 R)$. For $y \in O_{[\bar{\rho}, \rho]}$, we have $y=v+w$, where $\|v\| \leq R$, $w \in C_{K},\|v+w\| \in[\bar{\rho}, \rho]$, and hence $\|w\| \in[\bar{\rho}-R, \rho+R]$. Therefore $w \in I_{[\bar{\rho}-R, \rho+R]} \subset I_{[\bar{\rho}, \rho]}+B(0, R)$, and the result follows since $v \in B(0, R)$.

Now, take $z \in C_{K},\|z\|=1$, such that $B\left(z, \tau_{C_{K}}\right) \subset C_{K}$. Thus,

$$
B\left(\frac{(\bar{\rho}+\rho)}{2} z, \frac{(\bar{\rho}+\rho)}{2} \tau_{C_{K}}\right) \subset C_{K} \text {. }
$$


Observe that $\rho \geq 3 \bar{\rho}$ implies that $\bar{\rho} \leq \frac{(\bar{\rho}+\rho)}{2}-\frac{(\bar{\rho}+\rho)}{4}$ and $\frac{(\bar{\rho}+\rho)}{2}+\frac{(\bar{\rho}+\rho)}{4} \leq \rho$, so we have

$$
B\left(\frac{(\bar{\rho}+\rho)}{2} z, \frac{(\bar{\rho}+\rho)}{4} \tau_{C_{K}}\right)=B\left(\frac{(\bar{\rho}+\rho)}{2} z, 2 \kappa R\right) \subset I_{[\bar{\rho}, \rho]},
$$

where $\kappa=\frac{(\bar{\rho}+\rho)}{8} \frac{\tau_{C_{K}}}{R}$. Thus, $B(w, 2 R) \subset \frac{1}{\kappa} I_{[\bar{\rho}, \rho]}$ for $w=\frac{\bar{\rho}+\rho}{2 \kappa} z$.

$$
\begin{aligned}
\frac{\operatorname{Vol}\left(I_{[\bar{\rho}, \rho]}\right)}{\operatorname{Vol}\left(K_{[\bar{\rho}, \rho]}\right)} & \geq \frac{\operatorname{Vol}\left(I_{[\bar{\rho}, \rho]}\right)}{\operatorname{Vol}\left(O_{[\bar{\rho}, \rho]}\right)} \geq \frac{\operatorname{Vol}\left(I_{[\bar{\rho}, \rho]}\right)}{\operatorname{Vol}\left(I_{[\bar{\rho}, \rho]}+B(0,2 R)\right)} \\
& \geq \frac{\operatorname{Vol}\left(I_{[\bar{\rho}, \rho]}\right)}{\operatorname{Vol}\left(I_{[\bar{\rho}, \rho]}+\frac{1}{\kappa} I_{[\bar{\rho}, \rho]}\right)}=\frac{1}{\left(1+\frac{1}{\kappa}\right)^{n}} \geq\left(1-\frac{1}{\kappa}\right)^{n} .
\end{aligned}
$$

We will complete the proof in three steps. For $s \in[\bar{\rho}, \rho]$, consider the sets $I_{s}$ and $O_{s}$.

First note that

$$
\frac{\operatorname{Vol}_{n-1}\left(I_{s}\right)}{\operatorname{Vol}_{n-1}\left(O_{s}\right)}=\frac{\operatorname{Vol}_{n-1}\left(C_{K} \cap S^{n-1}\right)}{\operatorname{Vol}_{n-1}\left(C_{K}+B(0, R / s) \cap S^{n-1}\right)}=\frac{\operatorname{Vol}_{n-1}\left(C_{K} \cap S^{n-1}\right)}{\operatorname{Vol}_{n-1}\left(C_{K}+B(0, R / s) \cap S^{n-1}\right)}
$$

is a nondecreasing function of $s$. Second, observe that

$$
\frac{\operatorname{Vol}\left(I_{[\bar{\rho}, \rho]}\right)}{\operatorname{Vol}\left(O_{[\bar{\rho}, \rho]}\right)}=\frac{\int_{\bar{\rho}}^{\rho} \operatorname{Vol}_{n-1}\left(I_{s}\right) d s}{\int_{\bar{\rho}}^{\rho} \operatorname{Vol}_{n-1}\left(O_{s}\right) d s} .
$$

Next, we will make use of the following remark.

REMARK 3.1 For any $a<b$ and any two positive functions $g$ and $h$ such that

$$
\frac{g(s)}{h(s)} \text { is nondecreasing for } s \in[a, b] \text {, we have } \frac{g(a)}{h(a)} \leq \frac{\int_{a}^{b} g(s)}{\int_{a}^{b} h(s)} d s \leq \frac{g(b)}{h(b)} \text {. }
$$

Third, applying Remark 3.1 with $g(s)=\operatorname{Vol}_{n-1}\left(I_{s}\right), h(s)=\operatorname{Vol}_{n-1}\left(O_{s}\right), a=\bar{\rho}$ and $b=\rho$ to obtain

$$
P\left(X_{t} \in C_{K} \mid\left\|X_{t}\right\|=\rho\right)=\frac{\operatorname{Vol}_{n-1}\left(I_{\rho}\right)}{\operatorname{Vol}_{n-1}\left(K_{\rho}\right)} \geq \frac{\operatorname{Vol}_{n-1}\left(I_{\rho}\right)}{\operatorname{Vol}_{n-1}\left(O_{\rho}\right)} \geq \frac{\operatorname{Vol}\left(I_{[\bar{\rho}, \rho]}\right)}{\operatorname{Vol}\left(O_{[\bar{\rho}, \rho]}\right)} \geq\left(1-\frac{1}{\kappa}\right)^{n} .
$$

Finally, since $\bar{\rho}=\rho / 3$ we have $\kappa=\frac{\tau_{C_{K}} \rho}{6 R}$.

Proof. OF Remark 3.1. Simply note that $\frac{g(s)}{h(s)} \leq \frac{g(b)}{h(b)}$ for $s \in[a, b]$. Then

$$
\int_{a}^{b} g(s) d s=\int_{a}^{b} h(s) \frac{g(s)}{h(s)} d s \leq\left(\frac{g(b)}{h(b)}\right) \int_{a}^{b} h(s) d s,
$$

and a similar argument yields the lower bound.

Since the bound obtained in Lemma 3.2 is monotone in $\rho$ and trivially true for bounded convex sets $\left(\tau_{C_{K}}=0\right)$, we have the following useful corollary.

COROLlaRY 3.1 For any convex set $K$ and $\rho \geq 6 R / \tau_{C_{K}}$ we have

$$
P\left(X_{t} \in C_{K} \mid\left\|X_{t}\right\| \geq \rho\right) \geq\left(1-\frac{6 R}{\tau_{C_{K}} \cdot \rho}\right)^{n} .
$$

The previous results used conditioning on the event that the random points have large norm. It is natural to bound the probability of these conditional events as well.

Lemma 3.3 Suppose that $K$ is unbounded, and that $f_{t}$ is given by (1). For a random variable $X_{t}$ distributed according to $f_{t}$ we have

$$
P\left(\left\|X_{t}\right\| \geq \rho\right) \geq 1-4 e \rho t^{1 / p} .
$$


Proof. We can assume that $\rho t^{1 / p}<1 / 4 e$, since the bound is trivial otherwise. We use the notation introduced in the proof of Lemma $3.2, K_{[a, b]}=\{y \in K: a \leq\|y\| \leq b\}$.

Assuming that $K$ is unbounded, there exists $z \in C_{K},\|z\|=1$. Then,

$$
K_{[0, \rho]}+(\kappa+2 \rho) z \subset K_{[\kappa, \kappa+3 \rho]} \text { for any } \kappa \in \mathbb{R}_{+},
$$

since for each $x \in K_{[0, \rho]}$, we have

$$
\begin{aligned}
& \|x+(\kappa+2 \rho) z\| \leq\|x\|+(\kappa+2 \rho)\|z\| \leq \kappa+3 \rho, \\
& \|x+(\kappa+2 \rho) z\| \geq(\kappa+2 \rho)\|z\|-\|x\| \geq \kappa+\rho,
\end{aligned}
$$

and $x+(\kappa+2 \rho) z \in K$ (the latter follows from $\left.z \in C_{K}\right)$.

So, for any odd integer $m \geq 3, K_{[\rho, m \rho]}=\bigcup_{i=1}^{(m-1) / 2} K_{[(2 i-1) \rho,(2 i+1) \rho]}$, where the union is disjoint (except on a set of measure zero), and by (13) we have

$$
\operatorname{Vol}\left(K_{[\rho, m \rho]}\right)=\sum_{i=1}^{(m-1) / 2} \operatorname{Vol}\left(K_{[(2 i-1) \rho,(2 i+1) \rho]}\right) \geq \frac{(m-1)}{2} \operatorname{Vol}\left(K_{[0, \rho]}\right)
$$

Now, define $m:=\frac{1}{\rho t^{1 / p}}$. By assumption we have $\rho t^{1 / p}<1 / 4 e$, which implies $m \geq 4 e$ (again, we assume that $m$ in an odd integer for convenience). Thus,

$$
\begin{aligned}
P\left(\left\|X_{t}\right\| \geq \rho\right) & =\frac{\int_{K_{[\rho, \infty]}} e^{-t\|x\|^{p}} d x}{\int_{K_{[0, \rho]}} e^{-t\|x\|^{p} d x+\int_{K_{[\rho, \infty]}} e^{-t\|x\|^{p}} d x}} \\
& \geq \frac{\int_{K_{[\rho, m \rho]}} e^{-t\|x\|^{p}} d x}{\int_{K_{[0, \rho]}} e^{-t\|x\|^{p} d x+\int_{K_{[\rho, m \rho]}} e^{-t\|x\|^{p}} d x}} \\
& \geq \frac{\operatorname{Vol}\left(K_{[\rho, m \rho]}\right) e^{-t(m \rho)^{p}}}{\operatorname{Vol}\left(K_{[0, \rho]}\right)+\operatorname{Vol}\left(K_{[\rho, m \rho]}\right) e^{-t(m \rho)^{p}}} \\
& \geq \frac{(m-1) \operatorname{Vol}\left(K_{[\rho, m \rho]}\right) e^{-t(m \rho)^{p}}}{2 \operatorname{Vol}\left(K_{[\rho, m \rho]}\right)+(m-1) \operatorname{Vol}\left(K_{[\rho, m \rho]}\right) e^{-t(m \rho)^{p}}} \\
& \geq \frac{(m-1) e^{-t(m \rho)^{p}}}{2+(m-1) e^{-t(m \rho)^{p}}}
\end{aligned}
$$

Using the definition of $m$, we have

$$
\begin{aligned}
P\left(\left\|X_{t}\right\| \geq \rho\right) & \geq \frac{\left(\frac{1}{\rho t^{1 / p}}-1\right) e^{-1}}{2+\left(\frac{1}{\rho t^{1 / p}}-1\right) e^{-1}}=\frac{\left(\frac{1}{\rho t^{1 / p}}-1\right)}{2 e+\left(\frac{1}{\rho t^{1 / p}}-1\right)} \\
& \geq 1-\frac{2 e}{\frac{1}{\rho t^{1 / p}}-1} \quad=1-\frac{2 e \rho t^{1 / p}}{1-\rho t^{1 / p}} \geq 1-4 e \rho t^{1 / p} .
\end{aligned}
$$

Lemma 3.2 and Corollary 3.1 quantify the geometric notion mentioned earlier (motivated by Figure 2), that most points in $K$ outside a compact set are in $C_{K}$ if its width is positive. On the other hand, Lemma 3.3 shows that the norm of $X_{t}$ is likely to be greater than $0.01 / t^{1 / p}$. Taken together, they lead to a lower bound on the probability of the event $\left\{X_{t} \in C_{K}\right\}$.

TheOREm 3.2 Suppose that $K$ is unbounded, and $f_{t}$ is defined as in (1). Let $\delta \in(0,1)$ and suppose

$$
t \leq\left(\frac{\delta^{2} \tau_{C_{K}}}{96 e n R}\right)^{p}
$$

and let $X_{t}$ be a random variable distributed according to $f_{t}$. Then

$$
P\left(X_{t} \in C_{K}\right) \geq 1-\delta \text {. }
$$


Proof. All that is needed is to combine Lemmas 3.2 and 3.3 . Clearly,

$$
P\left(X_{t} \in C_{K}\right) \geq P\left(\left\|X_{t}\right\| \geq \rho\right) P\left(X_{t} \in C_{K} \mid\left\|X_{t}\right\| \geq \rho\right) .
$$

By Lemmas 3.2 and 3.3 , we have

$$
P\left(\left\|X_{t}\right\| \geq \rho\right) \geq 1-4 e \rho t^{1 / p} \text { and } P\left(X_{t} \in C_{K} \mid\left\|X_{t}\right\| \geq \rho\right) \geq\left(1-\frac{6 R}{\tau_{C_{K}} \cdot \rho}\right)^{n} .
$$

It is sufficient to ensure that

$$
1-4 e t \rho>1-\frac{\delta}{2} \quad \text { and } \quad\left(1-\frac{6 R}{\tau_{C_{K}} \cdot \rho}\right)^{n}>1-\frac{\delta}{2}
$$

for some $\rho \geq 6 R / \tau_{C_{K}}$. Noting that $(1-x)^{1 / n} \leq 1-\frac{x}{n}$ for all $x \in[0,1]$, the second relation also holds since if

$$
1-\frac{6 R}{\tau_{C_{K}} \cdot \rho}>1-\frac{\delta}{2 n} \text {. }
$$

It suffices to choose $\rho=\frac{12 n R}{\delta \tau_{C_{K}}}$ for the second relation to hold, and the first relation holds since

$$
4 e \rho t^{1 / p} \leq \frac{4 \delta^{2} \tau_{C_{K}} e 12 n R}{96 e n R \delta \tau_{C_{K}}}=\frac{\delta}{2} .
$$

Theorem 3.2 characterizes the behavior of random variables $X_{t} \sim f_{t}$ for values of $t$ that are "relatively small" with respect to its support $K$. It is natural to ask what kind of behavior one should expect for values of $t$ that are "relatively large" with respect to the support $K$. An answer to this question is given in the next lemma.

Lemma 3.4 Assume that there exists $\bar{v} \in K$ such that $\|\bar{v}\|=D$. If $t^{1 / p} \geq 1 / D$, then

$$
\max _{s \in S_{*}^{n-1}} P\left(\left|\left\langle s, X_{t}\right\rangle\right|>\frac{1}{4 e n t^{1 / p}}\right)>\frac{1}{9} .
$$

Proof. Let $\gamma=\frac{1}{4 e n t^{1 / p}}$. Since $D=\|\bar{v}\|=\max \left\{\langle s, \bar{v}\rangle: s \in B_{*}(0,1)\right\}$, there exists $v \in S_{*}^{n-1}$ such that $\langle v, \bar{v}\rangle=D$.

By obtaining a lower bound for $v$ we automatically obtain a lower bound for the maximum over the dual sphere. So,

$$
\max _{s \in S_{*}^{n-1}} P\left(\left|\left\langle s, X_{t}\right\rangle\right|>\gamma\right) \geq P\left(\left|\left\langle v, X_{t}\right\rangle\right|>\gamma\right) .
$$

It will be convenient to define the following sets

$$
A=\{x \in K:|\langle v, x\rangle|\langle\gamma\} \text { and } B=\{(1-\alpha) x+\alpha \bar{v}: x \in A\},
$$

where $\alpha=\gamma \frac{2}{D}<\frac{1}{2 e n}$.

Note that for any $y \in B, y \in K$ since $x$ and $\bar{v} \in K$ and $\alpha \in(0,1)$. Moreover,

$$
|\langle v, y\rangle| \geq\langle v, y\rangle=(1-\alpha)\langle v, x\rangle+\alpha\langle v, \bar{v}\rangle>-\gamma+\gamma \frac{2}{D} D=\gamma .
$$

Thus, $y \notin A$.

Next recall that for any $M>0$ (and $\|x\| \neq 0$ ), we have

$$
\|(1-\alpha) x+\alpha \bar{v}\|^{p} \leq\|x\|^{p}\left(1+\frac{2 \gamma}{M}\right)^{p}+(M+2 \gamma)^{p} .
$$


By choosing $M=2 e \gamma n$, we have

$$
\begin{aligned}
& P\left(X_{t} \in K \backslash A\right) \geq P\left(X_{t} \in B\right)=\int_{B} \frac{e^{-t\|y\|^{p}} d y}{\int_{K} e^{-t\|z\|} d z} \\
& =\frac{1}{\int_{K} e^{-t\|z\|^{p}} d z} \int_{A} e^{-t\|(1-\alpha) x+\alpha \bar{v}\|^{p}}(1-\alpha)^{n} d x \\
& \geq \frac{e^{-t(M+2 \gamma)^{p}}(1-\alpha)^{n}}{\int_{K} e^{-t\|z\|^{p}} d z} \int_{A} e^{-t\left\|\left(1+\frac{2 \gamma}{M}\right) x\right\|^{p}} d x \\
& \geq \frac{e^{-t(2 \gamma)^{p}(e n+1)^{p}}(1-\alpha)^{n}\left(1+\frac{1}{e n}\right)^{-n}}{\int_{K} e^{-t\|z\|^{p}} d z} \int_{\left(1+\frac{2 \gamma}{M}\right) A} e^{-t\|x\|^{p}} d x \\
& \geq e^{-t(2 \gamma)^{p} 4^{p} n^{p}} e^{-1 /(2 e-1)} e^{-1 / e} P\left(X_{t} \in A\right) \\
& \geq \frac{1}{e^{2}} P\left(X_{t} \in A\right)=\frac{1}{e^{2}}\left(1-P\left(X_{t} \in K \backslash A\right)\right)
\end{aligned}
$$

and we have $P\left(X_{t} \in K \backslash A\right) \geq 1 / 9$.

This result allows us to construct several useful bounds on the moments of $X_{t}$.

In the case of $p=1$, we can improve on Lemma 3.4 as follows.

COROLLARY 3.2 If in addition we have $p=1$, for $t \geq \sqrt{n} / D$ we have

$$
\max _{s \in S_{*}^{n-1}} P\left(\left|\left\langle s, X_{t}\right\rangle\right|>\frac{1}{4 e t \sqrt{n}}\right)>\frac{1}{3} .
$$

Proof. The proof is similar to Lemma 3.4. We define $A:=\left\{x \in K:|\langle v, x\rangle|<\frac{1}{4 e t \sqrt{n}}\right\}$, and $\alpha:=\frac{1}{2 e t D \sqrt{n}}<\frac{1}{2 e n}$. Next, note that the inequality (14) becomes

$$
\|(1-\alpha) x+\alpha \bar{v}\| \leq\|x\|+\alpha\|\bar{v}\|,
$$

and there is no need to use $M$. In this case, it follows that

$$
P\left(X_{t} \in K \backslash A\right) \geq e^{-t \alpha\|\bar{v}\|}(1-\alpha)^{n} P\left(X_{t} \in A\right) \geq \frac{1}{2} P\left(X_{t} \in A\right)=\frac{1}{2}\left(1-P\left(X_{t} \in K \backslash A\right)\right)
$$

and we have $P\left(X_{t} \in K \backslash A\right) \geq 1 / 3$. This holds since we have

$$
\begin{aligned}
e^{-t \alpha\|\bar{v}\|}(1-\alpha)^{n} & =e^{-1 /(2 e \sqrt{n})}\left(1-\frac{1}{2 e t D \sqrt{n}}\right)^{n} \geq e^{-1 / 2 e}\left(1-\frac{1}{2 e n}\right)^{n} \\
& \geq e^{-1 / 2 e} \cdot e^{-1 /(2 e-1)} \geq 0.664 \geq 1 / 2 .
\end{aligned}
$$

For the reader convenience, the next corollary is specialized for the case of $\|\cdot\|=\|\cdot\|_{2}$ and $p=1$; it will be used in the following section. It shows how one can relate the eigenvalues of the second moment matrix, the parameter $t$, and the diameter of $K$ (if the latter is bounded).

COROllary 3.3 Assume that $p=1$ and $\|\cdot\|=\|\cdot\|_{2}$. If $K$ is unbounded, then for every $t>0$

$$
\lambda_{\max }\left(E\left[X_{t} X_{t}^{\prime}\right]\right) \geq \frac{1}{n(7 e t)^{2}} .
$$

Otherwise, suppose that for some $t>0$ we have

$$
\lambda_{\max }\left(E\left[X_{t} X_{t}^{\prime}\right]\right)<\frac{1}{n(7 e t)^{2}} .
$$

Then $K$ is bounded and $K \subset B(0, \bar{R})$ for $\bar{R}=\frac{\sqrt{n}}{t}$. 
Proof. If one is using the Euclidean norm, we have $\|\cdot\|_{2}=\|\cdot\|=\|\cdot\|_{*}$. Moreover, we have that

$$
\lambda_{\max }\left(E\left[X_{t} X_{t}^{\prime}\right]\right)=\max _{s \in S^{n-1}}\left\langle s, E\left[X_{t} X_{t}^{\prime}\right] s\right\rangle=\max _{s \in S^{n-1}} E\left[\left\langle s, X_{t}\right\rangle^{2}\right] .
$$

If $K$ is unbounded, there exists $v \in K$ such that $\|v\| \geq \sqrt{n} / t$. Thus, we have

$$
\max _{s \in S^{n-1}} E\left[\left\langle s, X_{t}\right\rangle^{2}\right] \geq \frac{1}{n(4 e t)^{2}} \max _{s \in S^{n-1}} P\left(\left|\left\langle s, X_{t}\right\rangle\right|>\frac{1}{\sqrt{n}(4 e t)}\right) \geq \frac{1}{n(4 e t)^{2}} \frac{1}{3} \geq \frac{1}{n(7 e t)^{2}}
$$

where the second inequality follows from Corollary 3.2 .

For the second part, suppose there exists $\bar{v} \in K$, with $\|\bar{v}\| \geq \frac{\sqrt{n}}{t}$. Let $\bar{R}:=\|\bar{v}\| \geq \sqrt{n} / t$. Then $t \geq \sqrt{n} / \bar{R}$ and $\|\bar{v}\|=\bar{R}$, so by Corollary 3.2 we have a contradiction.

A similar result can be established for small values at $t$. Intuitively we will recover results known for the uniform density as we let $t$ goes to zero.

LEMMA 3.5 Assume that there exists $\bar{v} \in K$ such that $\|\bar{v}\|=D$. If $t^{1 / p}<1 /(2 D)$,

$$
\max _{s \in S_{*}^{n-1}} P\left(\left|\left\langle s, X_{t}\right\rangle\right|>\frac{D}{4 e n}\right)>\frac{1}{9} \text {. }
$$

Proof. The proof is similar to the proof of Lemma 3.4 if one defines

$$
A=\left\{x \in K:|\langle v, x\rangle|<\frac{D}{4 e n}\right\} .
$$

Corollary 3.4 Assume that $K$ has diameter $D$ and $t<1 /(2 D)$. Then,

$$
E\left[\left\|X_{t}\right\|\right] \geq \frac{D}{72 e n}
$$

Proof. If $K$ has diameter $D$, there exist two points $\bar{v}, \bar{w} \in K$ such that $\|\bar{v}-\bar{w}\|=D$. We can assume that $\|\bar{v}\| \geq D / 2$. The result follows from Lemma 3.5 .

We close this section with a simple observation with respect to the entropy of a density function

$$
\operatorname{Ent}(f)=-\int_{\mathbb{R}^{n}} f(x) \ln f(x) d x .
$$

COROLlary 3.5 If $f_{t}$ is a norm induced density function, then

$$
\operatorname{Ent}\left(f_{t}\right)=t E\left[\left\|X_{t}\right\|^{p}\right]
$$

Thus, Lemma 3.4, Lemma 3.5, and Corollary 3.2 can be used to bound the entropy.

ProOF. The result follows by noting that $-f(x) \ln f(x)=t\|x\|^{p} e^{-t\|x\|^{p}}$.

4. Testing the Boundedness of a Convex Set: a Density Homotopy. The algorithm we propose is a homotopy procedure to simulate a random variable which has desirable properties with respect to $K$. Motivated by the geometry of unbounded convex sets, the uniform density over $K$ would be an interesting candidate. Unfortunately, as opposed to most frameworks in the literature, a random variable which is uniformly distributed over $K$ will not be proper if $K$ is unbounded and cannot be used. Instead, we will work with a parameterized family of densities, $\mathcal{F}=\left\{f_{t}: t \in\left(0, t_{0}\right]\right\}$, such that $f_{t}$ is a proper density for every $t$. In addition, for any fixed compact subset of $K$ the parameterized density uniformly converges to the uniform density over that compact set as $t \rightarrow 0$. As mentioned earlier, the algorithm must provide us with a certificate of boundedness or unboundedness. Any nonzero element of the recession cone of $K$ is a valid certificate of unboundedness. We will assume that a membership oracle for the recession cone of $K$ itself is available. 
On the other hand, the certificate of boundedness is more thought-provoking. If $K$ is described by a set of linear inequalities, $K=\left\{x \in \mathbb{R}^{n}: A x \leq b\right\}, K$ will be bounded if and only if positive combinations of the rows of $A$ span $\mathbb{R}^{n}$. More generally, if $K$ is represented by a separation oracle, a valid certificate of boundedness would be a set of normal vectors associated with hyperplanes returned by the separation oracle whose positive combinations span $\mathbb{R}^{n}$. Note that a membership oracle provides much less information and we cannot sensibly extend the previous concept to our framework. Instead, our certificate of boundedness will be given by the eigenvalues of the second moment matrix associated with the random variables induced by the family $\mathcal{F}$. In contrast with the previous certificates, it will be a "probabilistic certificate of boundedness" since the true second moment matrix is unknown and must be estimated via a probabilistic method.

4.1 Assumptions and condition measures. In addition to Assumptions 3.1 and 3.2 , we make the following assumptions on the set $K$ :

Assumption $4.1 K$ is a closed convex set given by a membership oracle.

Assumption 4.2 There exists $r>0$ such that $B(0, r) \subseteq K$.

Assumption 4.3 A membership oracle is available for $C_{K}$, the recession cone of $K$.

The closedness of $K$ could be relaxed with minor adjustments on the implementation of the random walk. Assumption 4.3 specifies how $K$ is represented.

Assumption 4.2 is stronger than Assumption 3.1. It requires that we are given a point in the interior of $K$, which is assumed to be the origin without loss of generality. That is standard in the membership oracle framework, since the problem of finding a point in a convex set given only by a membership oracle is hard in general. Finally, we emphasize that only a lower bound on $r$ is required to implement our algorithm. Section 5.3 gives a simple procedure to obtain an approximation of $r$ within a factor of $\sqrt{n}$.

In our analysis, besides the dimension of $K$, there are three geometric quantities that naturally arise: $r, R$, and $\tau_{C_{K}}$. Not surprisingly, the dependence of the computational complexity of our algorithm on these geometric quantities differs if $K$ is bounded or unbounded (recall that the case of $\tau_{C_{K}}=0$ is fundamentally different if $K$ is bounded or unbounded). Nonetheless, in either case the dependence on these quantities will be only logarithmic. An instance of the problem is said to be ill-conditioned if $\tau_{C_{K}}=0$ and $K$ is unbounded, otherwise the instance is said to be well-conditioned.

4.2 The algorithm. In order to define the algorithm, let $f_{t}$ be defined as (1) with $\|\cdot\|=\|\cdot\|_{2}$ (the Euclidean norm). Let $\alpha \in(0,1)$ and let hit-and-run be a geometric random walk which will simulate the next random variable (see Section 6 for details). This yields the following "exact" method to test the boundedness of $K$ :

Density Homotopy Algorithm (Exact):

Input: $r$ such that $B(0, r) \subset K$, define $t_{0}=t_{\text {initial }}(r), \alpha \in(0,1)$, and set $k \leftarrow 0$.

Step 1. (Initialization) Draw $X_{t_{0}} \sim f_{t_{0}}(x)$.

Step 2. (Testing Unboundedness) If $X_{t_{k}} \in C_{K} \backslash\{0\}$, stop.

Step 3. (Variance and Mean) Compute the mean and covariance of $X_{t_{k}}: z_{k}$ and $V_{k}$.

Step 4. (Testing Boundedness) If $\lambda_{\max }\left(V_{k}+z_{k} z_{k}^{T}\right)<\frac{1}{n\left(7 e t_{k}\right)^{2}}$, stop.

Step 5. (Update Density) Update the parameter: $t_{k+1}=(1-\alpha) \cdot t_{k}$.

Step 6. (Random Point) Draw $X_{t_{k+1}} \sim \operatorname{hit-and-run}\left(f_{t_{k+1}}, X_{t_{k}}, V_{k}\right)$

Step 7. (Loop) Set $k \leftarrow k+1$, goto Step 2.

This (exact) method requires $r$, the exact draw of $X_{t_{k+1}}$, and the exact computation of the mean $z_{k+1}$ and covariance matrix $V_{k+1}$ of the random variable $X_{t_{k+1}}$. In order to obtain an implementable method, we can use only approximations of these objects.

Detailed bounds on the computational complexity of the hit-and-run procedure, on the estimation of $\hat{z}_{k+1}$ and $\hat{V}_{k+1}$, and $\hat{r}$ are provided in Sections 5.3, 6, and 7. Moreover, the use of an approximate mean and approximate covariance matrix must be taken into account in the test of boundedness (Step 4), which is presented in Theorem 4.1 . 
Each loop of the algorithm (Steps 2-7) is called an iteration of the algorithm. Thus, the work per iteration consists of (i) performing the hit-and-run random walk, (ii) computing an approximation of the covariance matrix, (iii) testing if the current point belongs to the recession cone, and (iv) computing the largest eigenvalue of a positive definite matrix. Although a highly accurate approximation of the covariance matrix is not needed, the probabilistic method used to estimate such matrix requires at least $O^{*}(n)$ samples. Such estimation will dominate the computational complexity per iteration.

Letting $t_{\text {final }}$ denote the final value of the parameter $t$ when the algorithm stops, the total number of iterations of the algorithm will be

$$
\left\lceil\frac{1}{\alpha} \cdot \ln \left(\frac{t_{\text {initial }}}{t_{\text {final }}}\right)\right\rceil
$$

The following theorem is the main characterizes the complexity of the homotopy algorithm.

TheOREm 4.1 Let $K$ be a convex set satisfying Assumptions 3.2, 4.2, 4.3, and 4.1 and consider the homotopy algorithm using a family of densities $\mathcal{F}=\left\{f_{t}(x) \sim 1_{K}(x) \cdot e^{-t\|x\|_{2}}: t \in\left(0, t_{0}\right]\right\}$. Then:

(i) If $K$ is unbounded, the algorithm will compute a valid certificate of unboundedness in at most

$$
O\left(\sqrt{n} \ln \left(\frac{n}{\delta} \frac{1}{\tau_{C_{K}}} \frac{R}{r}\right)\right) \quad \text { iterations with probability } 1-\delta
$$

where each iteration makes at most $O^{*}\left(n^{4} \ln \left(\frac{1}{\delta} \ln \left(\frac{1}{\delta} \frac{1}{\tau_{C_{K}}} \frac{R}{r}\right)\right)\right)$ calls to the membership oracle.

(ii) If $K$ is bounded, the algorithm will compute a valid certificate of boundedness in at most

$$
O\left(\sqrt{n} \ln \left(n \frac{R}{r}\right)\right) \text { iterations with probability } 1-\delta \text {, }
$$

where each iteration makes at most $O^{*}\left(n^{4} \ln \left(\frac{1}{\delta} \ln \left(\frac{R}{r}\right)\right)\right)$ calls to the membership oracle.

The proof of Theorem 4.1, which is provided in Section 10, is built upon the analysis of the next six sections.

\section{Analysis of the homothopy algorithm}

5.1 Stopping criteria: unbounded case. An appropriate certificate of unboundedness for a convex set is to exhibit a non-zero element of the recession cone of $K$. Assumption 4.3 allows us to correctly verify if a point is an element of $C_{K}$. For example, in the case of linear conic systems (3), any membership oracle for $C$ itself can be used to construct a membership oracle for $K$ and $C_{K}$.

If the algorithm terminates indicating that $K$ is unbounded, a nonzero element of the recession cone was found (a certificate of unboundedness). Thus, the algorithm always terminates correctly in this case. The following corollary of Theorem 3.2 ensures that we can find such certificate, which provides a desirable stopping criteria in the case of $K$ being unbounded.

Corollary 5.1 Suppose $K$ is unbounded. After

$$
T=\frac{1}{\alpha} \ln \left(t_{0} \frac{96 e n R}{\delta^{2} \tau_{C_{K}}}\right)
$$

iterations of the exact algorithm, we have $P\left(X_{t_{T}} \in C_{K}\right) \geq 1-\delta$.

Proof. We start the algorithm with $t=t_{0}$, and after $T$ iterations, we obtain $t_{T}=\frac{\delta^{2} \tau_{C_{K}}}{96 e n R}$. The result follows by applying Theorem 3.2 to $X_{t_{T}}$.

5.2 Stopping criteria: bounded case. In contrast with the unbounded case, we lack a straightforward certificate for the case of $K$ being bounded. In addition, an unbounded set whose recession cone has zero width should not be wrongly classified as bounded. That is, our analysis should cover such an ill-conditioned case as well. 
In the search for an appropriate certificate, the mean of the random variable $X_{t}$ appears as a natural candidate. Assuming that the set $K$ is unbounded and line-free, its norm should increase as the parameter $t$ decreases. On the other hand, if $K$ is bounded, the mean will remain bounded no matter how much $t$ decreases. Unfortunately, that analysis breaks down for sets that contain lines. For example, if $K$ is symmetric the mean of $X_{t}$ is zero for every $t>0$, whether $K$ is bounded or not.

In order to overcome this we consider the second moment matrix $\Omega_{t}$ of the random variable $X_{t}$. The matrix $\Omega_{t}$ will be large, in the positive definite sense, if either the covariance matrix or the norm of the mean of $X_{t}$ is large. Again, if $K$ is unbounded the maximum eigenvalue of $\Omega_{t}$ increases as the parameter $t$ decreases. Otherwise, $K$ being bounded, the maximum eigenvalue will eventually be bounded. This provides a nice criterion which is robust to instances where $K$ contains lines and/or $\tau_{C_{K}}=0$. We emphasize that the second order information is readily available, since we are required to compute the covariance matrix and the mean of $X_{t}$ to implement the hit-and-run random walk for $f_{t}$ (see Section $\underline{6}$ for details on the sampling theory and the reasons why we need to compute the covariance matrix to keep $f_{t}$ in near-isotropic position). The next corollary provides the desirable stopping criteria.

COROLlary 5.2 Suppose $K$ is bounded. The exact algorithm will detect boundedness for all $t<\frac{1}{7 e R \sqrt{n}}$, and will bound $R$ by $\frac{\sqrt{n}}{t}$. Moreover, this will happen in at most $T=\frac{1}{\alpha} \ln \left(t_{0} 7 e R \sqrt{n}\right)$ iterations.

Proof. If $t<\frac{1}{7 e R \sqrt{n}}$, then $\lambda_{\max }\left(E\left[X_{t} X_{t}^{\prime}\right]\right)<\frac{1}{(7 e t)^{2} n}$ from Corollary 3.3. From Corollary 3.3 , this means that $K$ is bounded and $R<\frac{\sqrt{n}}{t}$.

Moreover, starting with $t=t_{0}$, after $T=\frac{1}{\alpha} \ln \left(t_{0} 7 e R \sqrt{n}\right)$ iterations we obtain $t_{T} \leq \frac{1}{7 e R \sqrt{n}}$.

To generate a certificate of boundedness, the maximum eigenvalue of the second moment matrix must be estimated (Section 7 covers the necessary theory for that). Since it will be estimated via a probabilistic method, there is a probability of failure on each iteration which must be properly controlled (see Section 9 for details). Thus, in contrast to the unbounded case, if the algorithm terminates indicating that $K$ is bounded, there is a probability of failure associated with that decision which can be made smaller at the expense of computational time.

5.3 Initialization of the algorithm: unknown $\boldsymbol{r}$. This section clarifies how to start the algorithm, that is, how should we choose the initial value $t_{0}$ based on $r$. As is usual under the membership oracle framework, it is assumed that we know a point in the interior of $K$, which is taken to be the origin for convenience (Assumption 4.2). In some applications of interest such points are readily available, for example $0 \in$ int $K$ in the conic system $(\overline{3})$.

The implementation of Step 1 will be done by a simple accept-reject method, see [2]. Note that it is simple to draw a random variable $X_{t_{0}}$ whose density is proportional to $f_{t_{0}}(x) \sim e^{-t_{0}\left\|_{x}\right\|_{2}}$ on $\mathbb{R}^{n}$ instead of only on $K$ (pick a point uniformly on $S_{2}^{n-1}$ and then scale using a $\Gamma\left(n, t_{0}\right)$ random variable ${ }^{1}$ ). If it is the case that $X_{t_{0}} \in K$, we accept that point; otherwise, we draw another point according to $f_{t_{0}}$ and repeat the process until a point in $K$ is obtained.

Now, we need a constructive approach to bound $r$ from below. Fortunately, a simple procedure is available for estimating $r$ up to a factor of $\sqrt{n}$. That will be satisfactory since the final dependence on $r$ is only logarithmic. Consider the following quantity:

$$
\hat{r}=\min _{i=1, \ldots, n} \max \left\{t: t \mathbf{e}_{i} \in K, \quad-t \mathbf{e}_{i} \in K\right\},
$$

where $\mathbf{e}_{i} \in \mathbb{R}^{n}$ denotes the $i^{t h}$ unit vector of $\mathbb{R}^{n}$. It is clear that $\hat{r}$ can be approximated in $O\left(n\left|\ln \frac{1}{r}\right|\right)$ operations (via a simple binary search) and will not increase the order of the computational complexity of the algorithm. The next lemma provides a guarantee of the quality of the approximation provided by $\hat{r}$.

LEMMA 5.1 Let $r$ be the radius of the largest ball centered at the origin contained in $K$ and let $\hat{r}$ be as defined in (15). Then $\hat{r} \geq r \geq \hat{r} / \sqrt{n}$.

\footnotetext{
${ }^{1} \mathrm{~A} \Gamma(\alpha, \beta)$ random variable is characterized by the density $f(x)=\left(\beta^{\alpha} / \Gamma(\alpha)\right) x^{\alpha-1} e^{-\beta x}$ for $x \geq 0$, and zero otherwise.
} 
Proof. Clearly, $\hat{r} \geq r$. Note that $\hat{r} \mathbf{e}_{i} \in K$ for every $i$. Thus, the convex hull of these points contains a ball of radius $\hat{r} / \sqrt{n}$ which is contained in $K$. Therefore, $\hat{r} \geq r \geq \hat{r} / \sqrt{n}$.

We also need to ensure that the probability of the event $\left\{X_{t_{0}} \in K\right\}$ is reasonably large. The next lemma achieves this by a suitable choice of the initial parameter $t_{0}$ based on the radius $r$ of the largest ball centered at the origin contained in $K$.

Lemma 5.2 Assume that the ball centered at the origin with radius $r$ is contained in $K$. Let $X_{t_{0}}$ be a random variable whose density is proportional to $e^{-t_{0}\|x\|_{2}}$ for any $x \in \mathbb{R}^{n}$. Then if $t_{0} \geq 2 \frac{(n-1)}{r}$, we have

$$
P\left(X_{t_{0}} \in K\right) \geq 1-e^{n-\left(t_{0} r / 2\right)} .
$$

Proof. Using Lemma 5.16 of [1] ( since $t_{0} r /(n-1) \geq 2$ ) for the second inequality, we have

$$
\begin{aligned}
P\left(X_{t_{0}} \notin K\right) & \leq P\left(X_{t_{0}} \notin B(0, r)\right)=P\left(f_{t_{0}}\left(X_{t_{0}}\right) \leq f_{t_{0}}(0) e^{-t_{0} r}\right) \leq\left(e^{1-\frac{t_{0} r}{n-1}} \frac{t_{0} r}{n-1}\right)^{n-1} \\
& \leq e^{n-1}\left(e^{-\frac{t_{0} r}{n-1}} \frac{t_{0} r}{n-1}\right)^{n-1} \leq e^{n-\left(t_{0} r / 2\right)}
\end{aligned}
$$

since $e^{-c} c \leq e^{-c / 2}$ for every $c \geq 0$.

This allows us to efficiently implement the accept-reject method in Step 1 of the algorithm.

COROLlaRY 5.3 After computing $\hat{r}$ as in (15), it suffices to initialize the algorithm with

$$
t_{0}=\frac{8 n^{3 / 2}}{\hat{r}}
$$

to obtain that $P\left(X_{t_{0}} \in K\right) \geq 1-e^{-3 n}$.

Proof. Recall that Lemma 5.1 implies that $\hat{r} \geq r / \sqrt{n}$. By Lemma 5.2, $P\left(X_{t_{0}} \in K\right) \geq 1-$ $e^{n-4 n^{3 / 2} \frac{r}{\hat{r}}} \geq 1-e^{-3 n}$.

6. Sampling $f_{t}$ via a geometric random walk. The ability to sample according to any density in the family $\mathcal{F}$ is the driving force of our algorithm. Although a variety of densities can be perfectly simulated with negligible computational effort, that is no longer the case if we restrict the support of the density to be an arbitrary convex set $K$ given by a membership oracle. In fact, even to generate a random point distributed uniformly over a convex set is an interesting problem with many remarkable applications (linear programming, computing the volume, etc., see [6, [13, 10]).

Important tools to generate random points proportional to a density function restricted to a high dimensional convex set $K$ are the so-called geometric random walks. Starting with a point in $K$, at each step the random walk moves to a point according to some distribution that depends only (i) on the current point, and (ii) on the desired density $f$ to be simulated. Thus, the sequence of points of the random walk is a Markov chain whose state space is $K$. Moreover, there are simple choices of transition kernels (which is the continuous state space analog for the transition matrix for a finite state Markov chain) that make $f$ the unique stationary distribution of this Markov chain (for example, the celebrated Metropolis filter), which automatically ensures several asymptotic results for arbitrary Markov chains 2 . Going one step further, we are interested in the convergence rate to the stationary distribution, which is a much more challenging question (which could be arbitrarily slow in general). So we can bound the necessary number of steps required by the random walk to generate a random point whose density is approximately $f$.

By choosing $\mathcal{F}$ to be a family of logconcave densities (in our case the parameter $p=1$ ), we will be able to invoke several results from a recent literature which demonstrate the efficiency of one particular random walk called hit-and-run, see [8, 11, 12]. Roughly speaking, these results show that if (i) a relatively good approximation of the covariance matrix is available, and (ii) the density of the current point is close to the desired density, then only $O^{*}\left(n^{3}\right)$ steps of the random walk are necessary to generate a random point whose distribution is a good approximation of the desired (target) distribution. 
In our context, recall that the distribution of interest $f_{t_{k}}$ is changing at each iteration. The current approximation of the covariance matrix $\hat{V}_{k}$ will be used as the approximation to the true covariance matrix of the next iteration $V_{k+1}$, which in turn will be estimated by $\hat{V}_{k+1}$. In a similar way, the current point $X_{t_{k}}$, distributed approximately according to $f_{t_{k}}$, will be used as the starting point for the random walk to approximate a random variable distributed according to $f_{t_{k+1}}$. The parameter $\alpha$, which dictates the factor by which $t$ is decreased at each iteration, will be the largest value such that these approximations are valid from a theoretical perspective.

6.1 A geometric random walk: hit-and-run. There are several possible choices of geometric random walks. We refer to 16 for a recent survey. Here we use the so-called hit-and-run random walk. The implementation of this random walk requires the following as input: a density function $f$ (known up to a multiplicative constant), a starting point $X^{0}$, a covariance matrix $V$, and a number of steps $m$.

Subroutine: hit-and-run $\left(f, X^{0}, V, m\right)$

Step 0 Set $k \leftarrow 0$.

Step 1 Pick a random vector $d \sim N(0, V)$.

Step 2 Define the line $\ell\left(X^{k}, d\right)=\left\{X^{k}+t d: t \in \mathbb{R}\right\}$.

Step 3 Move to a point $X^{k+1}$ chosen according to $f$ restricted to $\ell\left(X^{k}, d\right)$.

Step 4 Set $k \leftarrow k+1$. If $k \leq m$, goto Step 1 .

Step 5 Report $X^{m}$.

Although hit-and-run can be implemented for arbitrary densities, we will restrict ourselves to the case of logconcave densities. In such case, the implementation of hit-and-run can be done efficiently, and we refer to the Appendix for a complete description for the case in which $f_{t}$ is defined as in (1) with $\|\cdot\|=\|\cdot\|_{2}$, the Euclidean norm, which is the proposed density for the algorithm.

6.2 Sampling complexity. Here we state without proof results in the literature of sampling random points according to logconcave densities. We start with a complexity result for the mixing time of the hit-and-run random walk.

ThEOREM 6.1 (10] Theorem 1.1) Let $f$ be a logconcave density and let $X$ denote the associated random variable. Moreover, $f$ is such that $(i)$ the level set of probability $1 / 8$ contains a ball of radius $s$, (ii) $E_{f}\left[\left\|X-z_{f}\right\|_{2}^{2}\right] \leq S^{2}$, and (iii) the $L_{2}$ norm of the starting distribution $\sigma$ with respect to the stationary distribution $\pi_{f}$ is at most $M$. Let $\sigma^{m}$ be the distribution of the current point after $m$ steps of hit-and-run applied to $f$ with $V=I$. Then, after

$$
m=O\left(\frac{n^{2} S^{2}}{s^{2}} \ln ^{5}\left(\frac{n M}{\varepsilon}\right)\right) \text { steps, }
$$

the total variation distance of $\sigma^{m}$ and $\pi_{f}$ is at most $\varepsilon$.

Theorem 6.1 bounds the rate of convergence of the geometric random walk not only by the dimension but also by the $L^{2}$ norm of the starting density with respect to the stationary density $f_{t}$, and by how "well-rounded" is $f_{t}$ via the ratio $S / s$. The notion of "well-rounded" is deeply connected with the concept of near isotropic position. The next lemma quantifies this connection.

Lemma 6.1 ([11] Lemma 5.13) Let $f$ be a density in $C$-isotropic position. Then $E_{f}\left[\left\|X-z_{f}\right\|_{2}^{2}\right] \leq C n$, and any upper level set $U$ of $f$ contains a ball of radius $\pi_{f}(U) / e \sqrt{C}$.

Any (full-dimensional) density can be put in near-isotropic position by a suitable linear transformation. By using an approximation of the covariance matrix $\hat{V}$ to implement the hit-and-run random walk such that all eigenvalues of $\hat{V}^{-1} V$ are between $1 / C$ and $C, f_{t}$ is in $C$-isotropic position. Thus, the ratio $S / s$ can be bounded by $8 e C \sqrt{n}$ (in this case, note that only $m=O^{*}\left(n^{3}\right)$ steps of the random walk will be necessary to generate one random sample). The next section summarizes how to compute an approximation $\hat{V}_{k}$ which puts $f_{t_{k}}$ in 2-isotropic position. Moreover, it will be shown that all densities simulated by the algorithm will be at most $C$-isotropic for a constant $C$ (independent of the dimension) as we decrease the homotopy parameter $t$. 
REMARK 6.1 In our analysis we will be assuming independence among different samples for simplicity (recall that they are separated by $m=O^{*}\left(n^{3}\right)$ steps of the random walk). Although this is not the case, independence can be approximated at the cost of an additional constant factor in the number $m$ of steps of the random walk. Here we have chosen exposition over formalism since no additional insight is gained if we work out all the details.

7. Estimating the covariance matrix, the mean, and the second moment matrix. In this section, we recall estimation results for the mean and covariance matrix of a logconcave random variable. Moreover, we show that these estimates can be used to approximate the second moment matrix with a desired relative accuracy. Herein it will be assumed that independent identically distributed samples $\left\{X^{i}\right\}$ are available. We emphasize that these estimations depend only on the samples and not on the isotropic position of the density function. As stated before, the isotropic position plays an important role to bound the number of steps of the chain required to obtain each sample.

First we recall a result for estimating the mean and covariance matrix.

LEMMA 7.1 ([11] Lemma 5.17) Let $z$ and $V$ denote respectively the mean and covariance matrix of a logconcave random variable. For any $\xi>0$ and $\delta \in(0,1)$, using

$$
N>\frac{4}{\xi^{2}} n \ln ^{2} \frac{1}{\delta} \quad \text { samples and } \hat{X}=\frac{1}{N} \sum_{i=1}^{N} X^{i}
$$

we have that

$$
P\left(\left\|V^{-1 / 2}(\hat{X}-z)\right\|_{2}>\xi\right) \leq \delta
$$

Lemma 7.2 ([6] Corollary A.2) Let $V$ denote the covariance matrix of a logconcave random variable. Using $N>O\left(\ln ^{3} \frac{1}{\delta} n \ln ^{2} n\right)$ samples, where $\delta<1 / n$, define

$$
\hat{V}=\frac{1}{N} \sum_{i=1}^{N}\left(X^{i}-\hat{X}\right)\left(X^{i}-\hat{X}\right)^{T}
$$

we have that all eigenvalues of the matrix $\hat{V}^{-1} V$ are in the interval $[1 / 2,2]$ with probability at least $1-\delta$.

These results yield a useful estimation procedure for the second moment matrix. In particular the maximum eigenvalue of $\Omega$ will be estimated up to a (known) constant factor.

LEMMA 7.3 Let $\Omega$ denote the second moment matrix associated with a logconcave random variable. Then for $\xi<1 / 4$ and using $N>O\left(\ln ^{3} \frac{1}{\delta} n \ln ^{2} n+\frac{1}{\xi^{2}} n \ln ^{2} \frac{1}{\delta}\right)$, for $\delta<1 / n$, the matrix

$$
\hat{\Omega}=\hat{V}+\hat{X} \hat{X}^{T}
$$

is such that all eigenvalues of $\hat{\Omega}^{-1} \Omega$ are in he interval $\left[(1 / 2-2 \xi),\left(2+2 \xi+\xi^{2}\right)\right]$ with probability at least $1-\delta$.

Proof. In this proof let $\|\cdot\|=\|\cdot\|_{2}$. Lemma 7.1 yields that $\left\|V^{-1 / 2}(\hat{X}-z)\right\| \leq \xi$ with probability greater than $1-\delta / 2$. In this event, there exists $d \in \mathbb{R}^{n}$ with $\|d\| \leq 1$ satisfying

$$
\hat{X}=z+\xi V^{1 / 2} d \text {. }
$$

For any $w \in \mathbb{R}^{n}$, we have that

$$
\begin{aligned}
\langle w, \hat{\Omega} w\rangle & =\langle w, \hat{V} w\rangle+\langle\hat{X}, w\rangle^{2}=\langle w, \hat{V} w\rangle+\left\langle z+\xi V^{1 / 2} d, w\right\rangle^{2} \\
& =\langle w, \hat{V} w\rangle+\langle z, w\rangle^{2}+2 \xi\langle z, w\rangle\left\langle d, V^{1 / 2} w\right\rangle+\xi^{2}\left\langle d, V^{1 / 2} w\right\rangle^{2} \\
& \geq \frac{1}{2}\langle w, V w\rangle+\frac{1}{2}\langle z, w\rangle^{2}-2 \xi\langle w, \Omega w\rangle=\left(\frac{1}{2}-2 \xi\right)\langle w, \Omega w\rangle .
\end{aligned}
$$

We will use the following corollary in our algorithm. 
Corollary 7.1 Using $N=O\left(n \ln ^{2} n \ln ^{3} \frac{1}{\delta}\right)$ samples to estimate the second moment matrix $\hat{\Omega}$, with probability at least $1-\delta$ we have that all eigenvalues of $\hat{\Omega}^{-1} \Omega$ are between $1 / 3$ and 3 .

ProOF. Set $\xi=1 / 24$ and apply Lemma 7.3 .

Thus, with $O^{*}(n)$ samples per iteration one can properly estimate the mean, covariance matrix, and second moment matrix to conduct the algorithm.

8. Updating the parameter $t$ : warm-start. Since the parameter $\alpha$ controls how fast the sequence $\left\{t_{k}\right\}$ decreases, its value is critical to the computational complexity of the algorithm. Although we are tempted to decrease $t$ as fast as possible, we still need to use the current density as a "warm-start" to approximate the next density. That is, the $L^{2}$ norm of $f_{t_{k}}$ with respect to $f_{t_{k+1}}$ needs to be controlled. Moreover, the covariance matrix associated with $f_{t_{k}}$ should also be close to the covariance matrix of the next iterate $f_{t_{k+1}}$. The trade-off among these quantities will decide how fast one can decrease the parameter $t$. Kalai and Vempala show how to relate the $L^{2}$ norm of two logconcave densities and their covariance matrices. For the reader's convenience we re-state their results here.

Lemma 8.1 ([6] Lemma 3.8) Let $f$ and $g$ be logconcave densities over $K$ with mean $z_{f}=E_{f}[X]$ and $z_{g}=E_{g}[X]$, respectively. Then, for any $c \in \mathbb{R}^{n}$

$$
E_{f}\left[\left\langle c, X-z_{f}\right\rangle^{2}\right] \leq 16\|f / g\| E_{g}\left[\left\langle c, X-z_{g}\right\rangle^{2}\right]
$$

This result implies that it suffices to control the $L^{2}$ norm of $f_{t_{k+1}}$ with respect to $f_{t_{k}}$ in order to guarantee that the covariance matrices of these densities are relatively close. A simple extension of Lemma 3.11 of [6] yields a bound this $L^{2}$ norm.

COROLlary 8.1 Consider the densities $f_{t_{k}}$ and $f_{t_{k+1}}$ such that $t_{k+1}=(1-\alpha) t_{k}=\left(1-\frac{1}{\sqrt{n}}\right) t_{k}$, i.e., $\alpha=1 / \sqrt{n}$. For $n \geq 5$,

$$
\left\|f_{t_{k}} / f_{t_{k+1}}\right\| \leq e^{n /(n-1)} \text { and }\left\|f_{t_{k+1}} / f_{t_{k}}\right\| \leq e^{n /(n-2 \sqrt{n})} .
$$

The next lemma combines the previous results to ensure that all the densities used in the algorithm will be in $2^{8}$-isotropic position.

Lemma 8.2 Assuming that $n \geq 16$, and using $N=O^{*}\left(\kappa^{3} n\right)$ samples at any iteration, the distribution encountered by the sampling algorithm in the next iteration is $2^{8}$-isotropic with probability $1-2^{-\kappa}$.

Proof. By using Lemma 7.2, we have that $f_{t_{k}}$ is 2-isotropic after we estimated its covariance matrix in Step 2 of the algorithm with probability $1-2^{-\kappa}$. Using Lemma 8.1 and Corollary 8.1, for any $v \in S^{n-1}$,

$$
E_{f_{t_{k+1}}}\left[\left\langle v, x-z_{f_{t_{k+1}}}\right\rangle^{2}\right] \leq 16 e^{n /(n-2 \sqrt{n})} E_{f_{t_{k}}}\left[\left\langle v, x-z_{f_{t_{k}}}\right\rangle^{2}\right] \leq 32 e^{n /(n-2 \sqrt{n})} \leq 2^{8},
$$

since $n \geq 16$.

Lemma 8.2 ensures that $O^{*}(n)$ samples suffice to estimate the current covariance matrix accurately enough to be used as an approximation to the covariance matrix associated with the next iteration.

9. Controlling the overall probability of failure. Before we proceed to the proof of Theorem 4.1, we prove a technical lemma which allows us to properly control the overall probability of failure of the algorithm. First note that if the algorithm finds an element of $C_{K}$ it will always stop correctly. Thus, the algorithm can fail only if it (wrongly) declares that $K$ is a bounded set or if it does not terminate. The latter concerns with the stopping criteria of the algorithm and it was already analyzed in Sections 5.1 and 5.2. The first issue can occur only if the estimated second moment matrix $\hat{\Omega}_{k}$ differs significantly from the true matrix $\Omega_{t_{k}}$. In turn their difference is controlled by the number of samples used to estimate $\hat{\Omega}_{t_{k}}$, which depends on the probability of failure used for the current iteration. Recall that we do not know the total number of iterations $T$ a priori (since we do not have any estimates for $R$ or $\tau_{C_{K}}$ ), and so we cannot simply set the probability of failure to $\delta / T$ for each iteration. Instead of using a constant 
probability of failure for all iterations, we slightly increase the number of samples used to estimate $\hat{\Omega}_{t_{k}}$ at each iteration to ensure that the probability of failure will not accumulate indefinitely.

LEMMA 9.1 Let $T$ be the total number of iterations of the algorithm and $1-\delta_{f}$ the desired probability of success. Initializing the algorithm with $\delta=\delta_{f} / 4$ and setting the probability of failure of the $i^{\text {th }}$ iteration to $\delta_{i}=\frac{1}{i^{2}} \delta_{f} / 4$, we obtain:

(i) the probability of success is at least $1-\delta_{f} / 2$;

(ii) The smallest $\delta$ used by the algorithm satisfies $\delta \geq \frac{\delta_{f}}{4} \frac{1}{T^{2}}$.

Proof. (i) By setting a probability of failure of the $i^{t h}$ iteration of the algorithm to $\left(1 / i^{2}\right) \delta_{f} / 4$, we have that the total probability of failure is bounded by

since $\sum_{i=1}^{\infty} \frac{1}{i^{2}}=\pi^{2} / 6<2$.

$$
\sum_{i=1}^{T} \delta_{i}=\sum_{i=1}^{T} \frac{\delta_{f}}{4 i^{2}} \leq \frac{\delta_{f}}{4} \sum_{i=1}^{\infty} \frac{1}{i^{2}} \leq \delta_{f} / 2
$$

(ii) follows since the algorithm terminates after $T$ iterations.

10. Proof of Theorem 4.1. Let $\alpha=\frac{1}{\sqrt{n}}$ and let $1-\delta_{f}$ be the desired probability of success. The algorithm terminates in $T=\sqrt{n} \ln \left(\frac{t_{\text {initial }}}{t_{\text {final }}}\right)$ iterations. Next we need to: $(i)$ properly bound $t_{\text {initial }},(i i)$ ensure that the algorithm terminates, i.e., properly bound $t_{\text {final }}$ (which also bounds $T$ ), and (iii) control the probability that it wrongly terminates.

(i) Corollary 5.3 yields $t_{\text {initial }}=t_{0}=8 n^{3 / 2} / \hat{r}$ which is at least $8 n / r$ and at most $8 n^{3 / 2} / r$.

(ii) We will use Corollaries 5.2 and 5.1 with $\delta=\delta_{f} / 2$. Thus, the algorithm will correctly terminate with probability at least $1-\delta_{f} / 2$ after at most

$$
\bar{T}=\sqrt{n} \ln \left(\left(8 n^{3 / 2} / r\right)(96 e n R) /\left(\left(\delta_{f} / 2\right)^{2} \tau_{C_{K}}\right)\right)
$$

iterations if $K$ is unbounded, and after at most

$$
\left.\bar{T}=\sqrt{n} \ln \left(\left(8 n^{3 / 2} / r\right) 21 e R \sqrt{n}\right)\right)
$$

iterations if $K$ is bounded (note that we use 21 instead of 7 to take into account a factor of 3 in the approximation of the maximum eigenvalue of $V_{k}$ ).

(iii) Finally, we ensure that the probability of (wrongly) terminating before reaching iteration $\bar{T}$ is at most $\delta_{f} / 2$. This is achieved by slightly reducing the probability of failure as described in Lemma 9.1,

Now we analyze the impact of reducing the probability of failure on the computational complexity of each iteration. Lemma $9.1(i i)$ ensures that it is sufficient to use

$$
\delta=\frac{\delta_{f} / 2}{4 \bar{T}^{2}}=\frac{\delta_{f}}{32 n \ln ^{2}\left(t_{\text {initial }} / t_{\text {final }}\right)} .
$$

This will dictate the number of samples needed to estimate the covariance matrix. In Lemma 8.2 we will need at most

$$
\kappa=\frac{1}{\ln 2} \ln \frac{32 \bar{T}^{2}}{\delta_{f}}
$$

at any given iteration (given that we terminate correctly). That leads to a total of at most

$$
O\left(n \ln ^{3}\left(\frac{\bar{T}}{\delta_{f}}\right)\right)
$$

samples per iteration of the algorithm where each sample requires $O^{*}\left(n^{3}\right)$ steps of the geometric random walk.

Defining a condition measure $\mu:=\frac{R}{r}$ if $K$ is bounded, or $\mu:=\frac{R}{r} \frac{1}{\tau_{C_{K}}}$ if $K$ is unbounded, the overall complexity is

$$
O^{*}\left(\bar{T} n^{4} \ln ^{3}\left(\frac{\bar{T}}{\delta_{f}}\right)\right)=O^{*}\left(n^{4.5} \ln \left(\frac{\mu}{\delta_{f}}\right) \ln ^{3}\left(\frac{n \ln (n \mu / \delta)}{\delta_{f}}\right)\right) .
$$

The results of Theorem 4.1 follow by using the appropriate $\bar{T}$ on each case as defined in (ii). 
11. Conclusions. In this work we study probability densities $f_{t}$ induced by an arbitrary norm with a convex support $K$ as defined by (1). Our goal is to relate geometric properties of $K$ with analytical properties of $f_{t}$.

Using these properties, we also develop an algorithm to test whether a convex set, given only by a membership oracle, is bounded or unbounded. The computational complexity of the probabilistic method proposed is polynomial in the dimension of the set and only logarithmic in other condition measures (such as the width of the recession cone and the diameter of the set, respectively, for the unbounded and bounded cases).

Clearly, one can derive a similar method based on uniform densities, instead of norm-induced densities, by defining $f_{t}(x) \sim 1_{K}(x) 1_{B(0,1 / t)}(x)$. These densities would be well-defined and many results of Section 3 can be adapted for this case as well. Although this could lead to a simpler analysis, the parameter $\alpha$ that is used to decrease $t$ on each iteration must be set to $1 / n$ instead of $1 / \sqrt{n}$ for the current density be a good "starting point" for the next density. This leads to an algorithm of the order $O^{*}\left(n^{5}\right)$.

Exploiting the geometry of unbounded convex sets was key in developing such probabilistic method. A concentration phenomenon guarantees that most points of an unbounded convex set will be in its recession cone if that cone has positive width. In such a case, random points with large norms are likely to belong to the recession cone which yields a certificate of unboundedness. In the case of the set being bounded, the second moment matrix associated with the random variable must also be bounded, in opposition to the unbounded case, which yields a certificate of boundedness.

In contrast with probabilistic methods over convex sets in the literature, we need to explicitly deal with unbounded sets, and additional effort is needed to ensure that all the densities are well defined. Moreover, if $K$ is unbounded our analysis shows that an element of $C_{K}^{\epsilon}$ can be computed in

$$
O^{*}\left(\sqrt{n} \ln \left(\frac{R}{r \epsilon}\right)\right) \text { iterations. }
$$

That is particularly relevant for the ill-conditioned case of $\tau_{C_{K}}=0$. Although we cannot find an element of $C_{K}$ (which has zero volume), the algorithm will generate a direction $d,\|d\|=1$, such that $\operatorname{dist}\left(d, C_{K}\right)<\epsilon$ in at most

iterations of the homotopy algorithm.

$$
O^{*}\left(\sqrt{n} \ln \left(\frac{R}{r} \frac{1}{\epsilon}\right)\right)
$$

Acknowledgment. I would like to thank Robert M. Freund, Santosh Vempala, Dimitris Bertsimas, Mahesh Nagarajan, Gustavo Didier, and Maurice Queyranne for many comments and suggestions that improved this work.

\section{References}

[1] D. Bertsimas and S. Vempala, Solving convex programs by random walks, Journal of the ACM 51 (2004), no. 4, 540-556.

[2] G. Casella and C. P. Robert, Monte carlo statistical methods, Springer, 1999.

[3] G. de Ghellink and J.-P. Vial, A polynomial Newtow method for linear programming, Algorithmica (1986), no. 1, 425-453.

[4] M. Gromov and V. Milman, Brunn Theorem and a concentration of volume of convex bodies, GAFA Seminar Notes, Tel Aviv University V (1984), 12pp.

[5] A. Iusem and A. Seeger, Axiomatization of the index of pointedness for closed convex cones, Mat. apl. comput. 24 (2005), no. 2, 245-283.

[6] A. Kalai and S. Vempala, Simulating annealing for convex optimization, to appear on Mathematic of Operations Research (2006).

[7] L. G. Khachiyan, Rounding of polytopes in the real number model of computation, Mathematics of Operations Research 21 (1996), no. 2, 307-320.

[8] L. Lovász, Hit-and-run mixes fast, Mathematical Programming 86 (1998), 443-461.

[9] L. Lovász and M. Simonovits, On the randomized complexity of volume and diameter, Proc. 33rd IEEE FOCS (1992), 482-491. 
[10] L. Lovász and S. Vempala, Fast algorithms for logconcave functions: Sampling, rounding, integration and optimization, Submitted. (2006).

[11] _ The geometry of logconcave functions and sampling algorithms, to appear in RSA (2006).

[12] _ _ Hit-and-run from a corner, SIAM Journal of Computing 35 (2006), no. 4, 985-100.

[13] _ Simulated annealing in convex bodies and an $o^{*}\left(n^{4}\right)$ volume algorithm, Journal of Computer and System Sciences 72 (2006), 392-417.

[14] Y. Nesterov, M. Todd, and Y. Ye, Infeasible-start primal-dual methods and infeasibility detectors for nonlinear programming problems, Mathematical Programming 84 (1999), 227-267.

[15] R. T. Rockafellar, Convex analysis, Princeton University Press, Princeton, New Jersey, 1970.

[16] S. Vempala, Geometric random walks: A survey, Combinatorial and Computational Geometry 52 (2005), 573-612.

Appendix A. Hit-and-run for $f(x) \sim e^{-\|x\|_{2}}$. Here we specialize the method for implementing the hit-and-run method for logconcave densities proposed in 11 to the densities defined as in (1) with the Euclidean norm.

Given $x_{0} \in K, d \in S^{n-1}$, define the line segment $\ell\left(x_{0}, d\right)=K \cap\left\{x_{0}+t d: t \in \mathbb{R}\right\}$ (note that if it is not a line segment, either $d$ or $-d$ belongs to $\left.C_{K}\right)$. The next iterate should be chosen from $\ell\left(x_{0}, d\right)$ according to the density $f$. Let $M_{\ell}=\max _{y \in \ell\left(x_{0}, d\right)} f(y)$ denote the maximum density on $\ell\left(x_{0}, d\right)$ and, for $v \in\left(0, M_{\ell}\right)$, let $L_{\ell}(v)$ denote the corresponding level set restricted to $\ell\left(x_{0}, d\right)$.

It is a two step method:

Step 1. Choose $v$ uniformly on $\left(0, M_{\ell}\right)$;

Step 2. Choose $y$ uniformly on $L_{\ell}(v)$.

To implement this, we need to compute three quantities: $M_{\ell}$ and the two endpoints of $L_{\ell}(v)$. It is possible to speed up the process by using closed-form formulas since we know the functional form of $f$ (although additional effort is necessary to adjust it for the support of $f$ ).

On $\ell\left(x_{0}, d\right), f$ can be written as $f\left(x_{0}+t d\right) \sim e^{-\sqrt{\left\|x_{0}\right\|^{2}+2 t\left\langle x_{0}, d\right\rangle+t^{2}\|d\|^{2}}}$. It is convenient to note that

$$
t^{*}=-\frac{\left\langle x_{0}, d\right\rangle}{\|d\|^{2}} \in \arg \max \left\{f\left(x_{0}+t d\right): t \in \mathbb{R}\right\},
$$

that is, for $x^{*}=x_{0}+t^{*} d, f\left(x^{*}\right) \geq M_{L}$. If $x^{*} \in K$, we have found $M_{\ell}$, otherwise, we need to make a binary search on $\left[x_{0}, x^{*}\right]$ to find it (note that in the second case we already have one of the endpoints of $\left.L_{\ell}(v)\right)$.

After drawing $v \in\left(0, M_{\ell}\right)$, again we can compute the explicit "unrestricted" point of where the endpoints should be

$$
\hat{t} \in\left\{t: v=e^{-\sqrt{\left\|x_{0}\right\|^{2}+2 t\left\langle x_{0}, d\right\rangle+t^{2}\|d\|^{2}}}\right\}
$$

or equivalently, the solution for the following quadratic equation

$$
\hat{t}^{2}\|d\|^{2}+2 \hat{t}\left\langle x_{0}, d\right\rangle+\left\|x_{0}\right\|^{2}+\ln ^{2} v=0 .
$$

Again, if the solutions $\hat{t}$ lie in $K$ we found an endpoint; otherwise, we need to perform binary search on $\left[x_{0}, x_{0}+\hat{t} d\right]$. 